\title{
INVERSE SPECTRAL PROBLEMS ON HYPERBOLIC MANIFOLDS AND THEIR APPLICATIONS TO INVERSE BOUNDARY VALUE PROBLEMS IN EUCLIDEAN SPACE
}

\author{
HIROSHI ISOZAKI
}

\begin{abstract}
We propose a new approach to solve the inverse boundary value problems in the Euclidean space. The idea consists in embedding the problem into hyperbolic manifolds and using their spectral properties. As a by-product, one can discuss the reconstruction of local conformal deformation of the metric of hyperbolic manifold from the spectral data at infinity. We also propose a new spectral data observed from the cusp neighborhood at infinity.
\end{abstract}

\section{INTRODUCTION}

There are two fundamental problems in the inverse spectral theory. One is the inverse boundary value problem $(I B V P)$. Let $\Omega$ be a bounded domain in $\mathbf{R}^{n}$ and consider the Dirichlet problem

$$
(-\Delta+V) u=0 \quad \text { in } \Omega, \quad u=f \quad \text { on } \quad \partial \Omega .
$$

The Dirichlet-Neumann map is defined by

$$
\Lambda_{V} f=\left.\frac{\partial u}{\partial \nu}\right|_{\partial \Omega},
$$

$\nu$ being the outer unit normal to the boundary. In IBVP, we try to reconstruct $V$ from $\Lambda_{V}$.

Another is the inverse scattering problem (ISP). For Schrödinger operastors $H_{0}=-\Delta, H=H_{0}+V$, define the wave operators $W_{ \pm}=\mathrm{s}-\lim _{t \rightarrow \pm \infty} e^{i t H} e^{-i t H_{0}}$, and the scattering operator $S=\left(W_{+}\right)^{*} W_{-}$. The S-matrix $S\left(\lambda ; \theta, \theta^{\prime}\right)$ is defined to be the integral kernel of $\mathcal{F} S \mathcal{F}^{*}$, where $\mathcal{F}$ is the Fourier tarnsformation and $\lambda$ is the energy parameter. The S-matrix $S\left(\lambda ; \theta, \theta^{\prime}\right)$ is observed in the behavior at infinity of solutions to the Schrödinger equation $(H-\lambda) \psi=0$. In fact if we put $A(\lambda ; \theta, \omega)=S(\lambda ; \theta, \omega)-\delta(\theta-\omega)$, we have

$$
\psi(x, \lambda, \omega) \sim e^{i \sqrt{\lambda} \cdot \omega x}-C(\lambda) \frac{e^{i \sqrt{\lambda} r}}{r^{(n-1) / 2}} A(\lambda ; \theta, \omega),
$$

as $r=|x| \rightarrow \infty, \theta=x / r$. In ISP, we try to reconstruct $V$ from the scattering matrix of $H$. We restrict ourselves here to the fixed energy problem, namely, the reconstruction of $V$ from the S-matrix of arbitrarily given fixed energy.

There is an extensive literature dealing with these subjects. For $n \geq 2$, these two problems are known to be equivalent and for $n \geq 3$, they are affirmatively solved by Sylvester-Uhlmann [36], Nachman [25], Khenkin-Novikov [19].

There is also an abundance of articles devoted to the spectral theory of Schrödinger operators on Riemannian manifolds (see e.g. Lax-Phillips [21], Agmon [1]). The uniqueness (up to diffeomorphism) of the metric with given D-N map was proved 
by Lee and Uhlmann [23], Sylvester [34] and Nachman [26]. Belishev and Kurylev constructed the Riemannian manifold itself from the boundary spectral data [2], [18]. However, the above mentioned problems in the fixed metric have not been considered yet.

For the moment, there is essentially only one method for solving IBVP and ISP. In IBVP it is called the method of complex geometrical optics, or exponentially growing solutions, and in ISP it is called Faddeev's Green function. These are two sides of one thing.

In this paper, we propose a new approach based on the spectral properties of hyperbolic manifolds, which we explain breifly here.

The fisrt fact we have to notice is:

The IBVP in the Euclidean space and that in the hyperbolic manifold are equivalent.

This can be easily observed in the 2-dimensional case. Multiplying the Schrödinger equation

$$
-\Delta u+q u=0
$$

in $\mathbf{R}^{2}$ by $x_{2}^{2}$, we have

$$
-x_{2}^{2} \Delta u+x_{2}^{2} q u=0
$$

which is just the Schrödinger equation in $\mathbf{H}^{2}$. Therefore the D-N maps $\widetilde{\Lambda}_{V}$ in $\mathbf{R}^{2}$ and $\Lambda_{x_{2}^{2} V}$ in $\mathbf{H}^{2}$ are related as follows

$$
\widetilde{\Lambda}_{V}=x_{2} \Lambda_{x_{2}^{2} V}
$$

In the general case, by putting $u=x_{n}^{(2-n) / 2} v$, we are led to the equation

$$
\left(-x_{n}^{2} \partial_{n}^{2}+(n-2) x_{n} \partial_{n}-x_{n}^{2} \Delta_{x}+V\right) v=0 \quad \text { in } \quad \Omega \subset \mathbf{H}^{n},
$$

where $V=x_{n}^{2} q-\frac{n(n-2)}{4}$, and the points in $\mathbf{H}^{n}$ are denoted as $\left(x, x_{n}\right), x_{n}>0$.

The next step is to use the gauge transformation $v=e^{i \theta \cdot x} u$ to introduce a parameter $\theta$ in the above equation :

$$
\left(-x_{n}^{2} \partial_{n}^{2}+(n-2) x_{n} \partial_{n}-x_{n}^{2}\left(\partial_{x}+i \theta\right)^{2}+V\right) u=0 \quad \text { in } \quad \Omega \subset \mathbf{H}^{n}, \quad \theta \in \mathbf{R}^{n-1} .
$$

In the 3rd step, we consider the action of simple discrete groups. We take a sufficiently large lattice $\Gamma$ of rank $n-1$ in $\mathbf{R}^{n-1}$ so that $\Omega$ is contained in one coordinate patch of the quotient space $\Gamma \backslash \mathbf{H}^{n}$. Then the above equation (1.3) can be regarded as the one in a domain in $\Gamma \backslash \mathbf{H}^{n}$. Here one should note that the operator $-x_{n}^{2} \partial_{n}^{2}+(n-2) x_{n} \partial_{n}+x_{n}^{2}\left(\partial_{x}+i \theta\right)^{2}$ is just the Floquet operator in the theory of periodic Schrödinger equation.

In the 4th step, we use the equivalence of IBVP and ISP in $\Gamma \backslash \mathbf{H}^{n}$ to construct the scattering matrix from the D-N map.

The final step is the complex Born approximation. By passing to the Fourier series, the Green's function of the Floquet operators are written by modified Bessel functions, $K_{i \sigma}\left(\zeta x_{n}\right), I_{i \sigma}\left(\zeta x_{n}\right), \zeta=\sqrt{\left(\gamma^{*}+\theta\right)^{2}}$. They are analytic with respect to $\theta$. Hence by varying $\theta$ along the imaginary axis, one can recover the potential $q$ from the scattering matrix. This procedure works for $n \geq 3$.

In summary, one can show the following theorem. 
Let $\mathcal{M}$ be an $n$-dimensional hyperbolic manifold with $n \geq 3$, and $\Omega$ a contractible bounded open set in $\mathcal{M}$ with smooth boundary. Suppose 0 is not a Dirichlet eigenvalue of $-\Delta_{g}+V$. Then $V$ is uniquely reconstructed from the $D-N$ map.

In fact $\mathcal{M}=\Gamma \backslash \mathbf{H}^{n}$ for some discrete group $\Gamma$. Hence by passing to the universal covering space, we see that the Dirichlet problem on a bounded contractible open set in $\mathcal{M}$ is equivalent to that on a similar set in $\mathbf{H}^{n}$.

By examining the above procedures, one can introduce a new notion of spectral data, the scattering amplitude associated with the cusp neighborhood at infinity. One can show that

Let $n \geq 2$. Suppose that $\Gamma \backslash \mathbf{H}^{n}$ has a cusp and that $-\Delta_{g}$ is perturbed in a contractible compact set in $\Gamma \backslash \mathbf{H}^{n}$. Then to know the associated $D-N$ map is equivalent to know the scattering amplitude at the cusp.

Using this fact, one can reconstruct the conformal perturbation of the metric for $n \geq 3$ and the general perturbation of the metric modulo conformal deformation for $n=2$ from the scattering amplitude at the cusp of $\Gamma \backslash \mathbf{H}^{n}$. This fact also holds for the arithmetic surface $S L(2, \mathbf{Z}) \backslash \mathbf{H}^{2}$.

Although our method is apparently very different from the usual ones, there is a close connection between our approach and the methods already established for the multi-dimensional inverse problems. The crucial trick in this paper is to consider the analytic continuation with respect to the Floquet parameter $\theta$ of the resolvent $R_{0}(\lambda+i 0 ; \theta)$ (see $\S 5$ ). This idea is inspired by the direction dependent Green operator of Faddeev ([6], [25], [19]). Employing the family of scattering amplitudes for the Floquet operators as the spectral data avoids the difficulty of exceptional points, the main barrier of the inverse scattering problem. This is analogus to the situation in the approach of Eskin-Ralston ([5], [12]). Our reconstruction procedure is the complex Born approximation, the method frequently used in the inverse scattering theory.

To study the continuous spectrum of the Schrödinger operator, the first important step is the limiting absorption principle (LAP). Basically there are three methods for the resolvent estimates ; the framework of scattering metric (Melrose [24]), the commutator method based on the Mourre inequality (Froese-Hislop [7], Hislop [8]), and the methods of a-priori estimates (Perry [28]). In this paper we adopt a more classical approach based on integration by parts developed by IkebeSaito [9]. This will make the argument self-contained except for $\S 9$, in which we need word by word translation of a part of the work of Saito [30]. Let us remark that if the potential is compactly supported, the contents of $\S 9$ can be replaced by an elementary argument using the properties of Bessel functions.

The plan of this paper is as follows. In $\S 2$, we prepare basic properties of Bessel functions. We study spectral properties of Schrödinger operators on $\mathbf{H}^{n}$ and $\Gamma \backslash \mathbf{H}^{n}$ in $\S 3$ and $\S 4$, leaving the proof in later sections. In $\S 5$, we reconstruct the Fourier coefficients of the potential from the scattering amplitudes of Floquet operators on $\Gamma \backslash \mathbf{H}^{n}$. In $\S 6$, we study the relation between ISP and IBVP in $\Gamma \backslash \mathbf{H}^{n}$, and derive the main theorem on IBVP in $\mathbf{R}^{n}$. We shall prove in $\S 7$ LAP for the 1-dimensional operator $-\partial_{y}^{2}+e^{2 y} \lambda$, which is utilized in $\S 8$ to prove LAP for Schrödinger operators on $\mathbf{H}^{n}$ and $\Gamma \backslash \mathbf{H}^{n}$. In $\S 9$ we state an abstract version of the theorem due to Saito [30] on the growth properties of solutions to the ordinary differential equation with 
operator-valued coefficients. In $\S 10$, we shall discuss the scattering amplitude at the cusp for general hyperbolic manifold and reconstruction of the metric. More detailed arguments of this section are given in [13]. We also note that in [14] an application of this hyperbolic space approach is given to derive local properties of Dirichlet-to-Neumann map for the inverse boundary value problem in the Euclidean space.

The notation in this paper is almost standard. $C^{\prime}$ s denote various constants. For two Banach spaces $X$ and $Y, \mathbf{B}(X ; Y)$ denotes the totality of bounded operators from $X$ to $Y$, and $\mathbf{B}(X)=\mathbf{B}(X ; X)$. For $x \in \mathbf{R}^{n},\langle x\rangle=\left(1+|x|^{2}\right)^{1 / 2}$. For $\zeta=\left(\zeta_{1}, \cdots, \zeta_{n}\right) \in \mathbf{C}^{n}, \zeta^{2}=\sum_{j=1}^{n} \zeta_{j}^{2}$. We denote by $L^{2}(\Omega ; d \mu)$ the $L^{2}$-space over $\Omega$ with respect to the measure $d \mu$. The spectrum of a closed operator $A$ is denoted by $\sigma(A)$. In sections 7 and 8 , we use a non-standard notation. For a set $\{\cdots\}, F(\cdots)$ denotes its characteristic function. For example $F(y<0)$ means the characteristic function of the set $\{y \in \mathbf{R} ; y<0\}$ in $\S 7$, while in $\S 8$ it will mean that of $\{(x, y) ; x \in \mathbf{E}, y<0\}$ or that of $\left\{(x, y) \in \mathbf{R}^{n} ; y<0\right\}$. There will be no fear of confusion.

The author is indebted to James Ralston for pointing out errors in the earlier version of this paper. He also thanks to Gunther Uhlmann for useful discussions and encouragements.

\section{BeSSEL FUnCtions}

2.1 Bessel functions. Let $J_{\nu}(x)$ be the Bessel function of order $\nu \notin \mathbf{Z}$, and for $x>0$ let

$$
I_{\nu}(x)=e^{-\nu \pi i / 2} J_{\nu}(i x), \quad K_{\nu}(x)=\frac{\pi}{2} \frac{I_{-\nu}(x)-I_{\nu}(x)}{\sin (\nu \pi)}
$$

be modified Bessel functions. They are analytic in the complex plane with cut along the negative real axis and

$$
I_{\nu}(z)=\left(\frac{z}{2}\right)^{\nu} \sum_{k=0}^{\infty} \frac{\left(z^{2} / 4\right)^{k}}{k ! \Gamma(k+\nu+1)} .
$$

By (2.2), for $x>0, \sigma \in \mathbf{R}$,

$$
\overline{I_{i \sigma}(x)}=I_{-i \sigma}(x), \quad \overline{K_{i \sigma}(x)}=K_{-i \sigma}(x)=K_{i \sigma}(x) .
$$

One also has for $0<x<1, \sigma \neq 0$,

$$
\left|I_{i \sigma}(x)\right| \leq C x^{-\operatorname{Im} \sigma}, \quad\left|K_{i \sigma}(x)\right| \leq C x^{-|\operatorname{Im} \sigma|} .
$$

The following asymptotic expansion is well-known :

$$
\begin{gathered}
I_{\nu}(z) \sim \frac{e^{z}}{\sqrt{2 \pi z}}+\frac{e^{-z+(\nu+1 / 2) \pi i}}{\sqrt{2 \pi z}}, \quad|z| \rightarrow \infty, \quad-\frac{\pi}{2}<\arg z<\frac{3 \pi}{2}, \\
I_{\nu}(z) \sim \frac{e^{z}}{\sqrt{2 \pi z}}+\frac{e^{-z-(\nu+1 / 2) \pi i}}{\sqrt{2 \pi z}}, \quad|z| \rightarrow \infty, \quad-\frac{3 \pi}{2}<\arg z<\frac{\pi}{2}, \\
K_{\nu}(z) \sim \sqrt{\frac{\pi}{2 z}} e^{-z}, \quad|z| \rightarrow \infty, \quad-\pi<\arg z<\pi .
\end{gathered}
$$

In particular

$$
K_{\nu}(-i x) \sim \sqrt{\frac{\pi}{2 x}} e^{i(x+\pi / 4)}, \quad x \rightarrow \infty
$$


(See e.g. [39] p. 202.) The following formulas are known as Kontorovich-Lebedev inversion formulas

$$
\begin{aligned}
& \frac{1}{\pi^{2}} \int_{0}^{\infty} \frac{K_{i \sigma}(x) K_{i \sigma}(y)}{\sqrt{x y}} 2 \sigma \sinh (\pi \sigma) d \sigma=\delta(x-y), \\
& \frac{2 \sigma \sinh (\pi \sigma)}{\pi^{2}} \int_{0}^{\infty} \frac{K_{i \sigma}(x) K_{i \tau}(x)}{x} d x=\delta(\sigma-\tau) .
\end{aligned}
$$

(See e.g. [37] pp.138, 145.)

2.2 Auxiliary operator. We reduce the analysis of our Schrödinger operators on hyperbolic manifolds to that of the operator

$$
L_{0}(\zeta)=-\partial_{y}^{2}+e^{2 y} \zeta^{2}
$$

in $L^{2}(\mathbf{R} ; d y)$ with parameter $\zeta \in \mathbf{C}$. Here $\partial_{y}=d / d y$. If $\zeta \geq 0,\left.L_{0}(\zeta)\right|_{C_{0}^{\infty}(\mathbf{R})}$ is essentially self-adjoint, whose self-adjoint realization is also denoted by $L_{0}(\zeta)$. For $s \in \mathbf{R}$, we introduce the weighted $L^{2}$-space $L^{2, s}=L^{2, s}\left(\mathbf{R}^{1}\right)$ by

$$
u \in L^{2, s} \Longleftrightarrow\|u\|_{s}^{2}=\int_{-\infty}^{\infty}(1+|y|)^{2 s}|u(y)|^{2} d y<\infty .
$$

We shall prove in $\S 7$ that for $\zeta \geq 0, E>0$ and $s>1 / 2$, there exist the following strong limits

$$
\mathrm{s}-\lim _{\epsilon \downarrow 0}\left(L_{0}(\zeta)-(E \pm i \epsilon)\right)^{-1}=:\left(L_{0}(\zeta)-E \mp i 0\right)^{-1} \in \mathbf{B}\left(L^{2, s} ; L^{2,-s}\right) .
$$

2.3 Green function. For $\zeta, \sigma \in \mathbf{C}$ with $\zeta \neq 0$, the equation

$$
\left(L_{0}(\zeta)-\sigma^{2}\right) u=0
$$

has two linearly independent solutions

$$
K_{i \sigma}\left(\zeta e^{y}\right), \quad I_{i \sigma}\left(\zeta e^{y}\right) .
$$

In the following, we always assume that $\operatorname{Re} \zeta \geq 0$. We put

$$
G\left(y, y^{\prime} ; \sigma, \zeta\right)= \begin{cases}K_{i \sigma}\left(\zeta e^{y}\right) I_{i \sigma}\left(\zeta e^{y^{\prime}}\right), & y>y^{\prime} \\ I_{i \sigma}\left(\zeta e^{y}\right) K_{i \sigma}\left(\zeta e^{y^{\prime}}\right), & y^{\prime}>y\end{cases}
$$

By $(2.4) \sim(2.7)$, it satisfies for $\sigma \in \mathbf{R} \backslash\{0\}$

$$
\left|G\left(y, y^{\prime} ; \sigma, \zeta\right)\right| \leq \frac{C \exp \left(-\operatorname{Re} \zeta\left|e^{y}-e^{y^{\prime}}\right|\right)}{\left(1+\left|\zeta e^{y}\right|^{1 / 2}\right)\left(1+\left|\zeta e^{y^{\prime}}\right|^{1 / 2}\right)},
$$

where $C$ is a constant independent of $y, y^{\prime}, \zeta$. We shall use this estimate in the following two ways :

$$
\begin{gathered}
\left|G\left(y, y^{\prime} ; \sigma, \zeta\right)\right| \leq C, \\
\left|G\left(y, y^{\prime} ; \sigma, \zeta\right)\right| \leq C|\zeta|^{-1} e^{-\left(y+y^{\prime}\right) / 2} .
\end{gathered}
$$

For $f \in C_{0}^{\infty}(\mathbf{R})$, we define $G(\sigma, \zeta) f$ by

$$
G(\sigma, \zeta) f=\int_{-\infty}^{\infty} G\left(y, y^{\prime} ; \sigma, \zeta\right) f\left(y^{\prime}\right) d y^{\prime} .
$$

Then $u=G(\sigma, \zeta) f$ satisfies

$$
\left(L_{0}(\zeta)-\sigma^{2}\right) u=f
$$

If $\zeta>0$ and $\operatorname{Im} \sigma<0, G(\sigma, \zeta) f \in L^{2}(\mathbf{R})$ by (2.4). Therefore

$$
G(\mp \sqrt{E \pm i \epsilon}, \zeta)=\left(L_{0}(\zeta)-(E \pm i \epsilon)\right)^{-1} \text {. }
$$


In particular

$$
G(\mp \sqrt{E}, \zeta)=\left(L_{0}(\zeta)-E \mp i 0\right)^{-1}, \quad E>0, \quad \zeta>0 .
$$

2.4 Spectral representation. Let $f \in L^{2, s}, s>1 / 2$. Since

$$
I_{\nu}(z) \sim \frac{1}{\Gamma(1+\nu)}\left(\frac{z}{2}\right)^{\nu} \quad(z \rightarrow 0),
$$

we have for $\sigma \in \mathbf{R} \backslash\{0\}, \zeta>0$ and $y \rightarrow-\infty$

$$
G(\sigma, \zeta) f \sim \frac{1}{\Gamma(1+i \sigma)}\left(\frac{\zeta}{2}\right)^{i \sigma} e^{i \sigma y} \int_{-\infty}^{\infty} K_{i \sigma}\left(\zeta e^{t}\right) f(t) d t .
$$

Let $K(\zeta)$ be the operator defined by

$$
(K(\zeta) f)(\sigma)=\frac{1}{\pi} \int_{-\infty}^{\infty} K_{i \sigma}\left(\zeta e^{y}\right) f(y) d y .
$$

It follows from (2.1) and (2.3) that the integral kernel of $\frac{1}{2 \pi i}[G(-\sqrt{E}, \zeta)-G(\sqrt{E}, \zeta)]$ is

$$
\frac{\sinh (\sqrt{E} \pi)}{\pi^{2}} K_{i \sqrt{E}}\left(\zeta e^{y}\right) K_{i \sqrt{E}}\left(\zeta e^{y^{\prime}}\right) .
$$

This implies that for $f \in C_{0}^{\infty}(\mathbf{R}), \zeta>0, E>0$

$$
\begin{gathered}
\frac{1}{2 \pi i}\left(\left[\left(L_{0}(\zeta)-E-i 0\right)^{-1}-\left(L_{0}(\zeta)-E+i 0\right)^{-1}\right] f, f\right) \\
=|(K(\zeta) f)(\sqrt{E})|^{2} \sinh (\sqrt{E} \pi) .
\end{gathered}
$$

Therefore by the standard argument from spectral theory and the formulas (2.9), (2.10), one can show that

$$
K(\zeta): L^{2}\left(\mathbf{R}^{1} ; d y\right) \rightarrow L^{2}((0, \infty) ; 2 \sigma \sinh (\pi \sigma) d \sigma)
$$

is unitary and

$$
\left(K(\zeta) L_{0}(\zeta) f\right)(\sigma)=\sigma^{2}(K(\zeta) f)(\sigma) .
$$

In contrast to (2.25), if we let $y \rightarrow \infty$ in $G(\sigma, \zeta) f(y)$ for $\sigma \in \mathbf{R} \backslash\{0\}, \zeta>0$ and compactly supported $f$, we have

$$
G(\sigma, \zeta) f \sim \sqrt{\frac{\pi}{2 \zeta}} \exp \left(-\frac{y}{2}-\zeta e^{y}\right) \int_{-\infty}^{\infty} I_{i \sigma}\left(\zeta e^{t}\right) f(t) d t .
$$

\section{Schrödinger OPERATORS ON $\mathbf{H}^{n}$}

3.1 Basic spectral properties. Let $\mathbf{H}^{n}=\mathbf{R}_{+}^{n}=\left\{\left(x, x_{n}\right) ; x \in \mathbf{R}^{n-1}, x_{n}>0\right\}$ be the hyperbolic space equipped with the metric $g=d s^{2}=x_{n}^{-2}\left((d x)^{2}+\left(d x_{n}\right)^{2}\right)$. The Laplace-Beltrami operator on $\mathbf{H}^{n}$ is

$$
\Delta_{g}=x_{n}^{2} \partial_{n}^{2}-(n-2) x_{n} \partial_{n}+x_{n}^{2} \Delta_{x},
$$

where $\partial_{n}=\partial / \partial x_{n}, \Delta_{x}=\sum_{i=1}^{n-1}\left(\partial / \partial x_{i}\right)^{2}$. By the change of variable $y=\log x_{n}$ and the unitary operator

$$
L^{2}\left(\mathbf{H}^{n} ; x_{n}^{-n} d x d x_{n}\right) \ni u \rightarrow v=e^{-(n-1) y / 2} u \in L^{2}\left(\mathbf{R}^{n} ; d x d y\right),
$$

the equation

$$
\left(-\Delta_{g}-\frac{(n-1)^{2}}{4}-z\right) u=f
$$


is transformed into

$$
\begin{gathered}
\left(H_{0}-z\right) v=e^{-(n-1) y / 2} f, \\
H_{0}=-\partial_{y}^{2}-e^{2 y} \Delta_{x} .
\end{gathered}
$$

Let $H=H_{0}+V$, where $V=V_{1}(x, y)+V_{2}(x, y)$ is a real function on $\mathbf{R}^{n}$. We shall assume that $V_{1}$ is compactly supported and

$$
V_{1}(x, y) \in L^{p}\left(\mathbf{R}^{n}\right)
$$

where $p=2$ for $n \leq 3, p>n / 2$ for $n \geq 4$,

$$
\left|V_{2}(x, y)\right| \leq C(1+|x|)^{-\rho}(1+|y|)^{-1-\rho}
$$

for some constants $C, \rho>0 .\left.H\right|_{C_{0}^{\infty}\left(\mathbf{R}^{n}\right)}$ is essentially self-adjoint, whose self-adjoint extension is denoted by $H$ also. Let $R(z)=(H-z)^{-1}$, and $R_{0}(z)=\left(H_{0}-z\right)^{-1}$. For $t, s \in \mathbf{R}$, let $\mathcal{H}^{t, s}$ be the weighted Hilbert space endowed with the norm

$$
\|u\|_{t, s}=\left\|(1+|x|)^{t}(1+|y|)^{s} u(x, y)\right\|_{L^{2}\left(\mathbf{R}^{n} ; d x d y\right)}<\infty .
$$

The following theorem will be proved in $\S 8$.

Theorem 3.1. (1) $\sigma_{e}(H)=[0, \infty)$.

(2) $\sigma_{p}(H) \cap(0, \infty)=\emptyset$.

(3) For $0<\delta<\rho, s>1 / 2$ and $\lambda>0$, there exist the strong limits

$$
\mathrm{s}-\lim _{\epsilon \downarrow 0} R(\lambda \pm i \epsilon)=: R(\lambda \pm i 0) \in \mathbf{B}\left(\mathcal{H}^{0, s} ; \mathcal{H}^{-\delta,-s}\right) .
$$

(4) For $s>1 / 2$ and $\lambda>0$, there exist the weak limits

$$
\mathrm{w}-\lim _{\epsilon \downarrow 0} R_{0}(\lambda \pm i \epsilon)=: R_{0}(\lambda \pm i 0) \in \mathbf{B}\left(\mathcal{H}^{0, s} ; \mathcal{H}^{0,-s}\right) .
$$

3.2 Spectral representation for $H_{0}$. Let $F_{0}$ be the Fourier transformation on $\mathbf{R}^{n-1}$ :

$$
F_{0} u(\xi)=(2 \pi)^{-(n-1) / 2} \int_{\mathbf{R}^{n-1}} e^{-i x \cdot \xi} u(x) d x .
$$

Let $R_{0}(z)=\left(H_{0}-z\right)^{-1}$. Then by $(2.11)$

$$
R_{0}(z)=F_{0}^{-1}\left(L_{0}(|\xi|)-z\right)^{-1} F_{0} .
$$

The right-hand side should be written as $\left(F_{0} \otimes 1\right)^{-1} 1 \otimes\left(L_{0}(|\xi|)-z\right)^{-1}\left(F_{0} \otimes 1\right)$. However, we employ the abbreviation of writing $A B$ instead of $(A \otimes 1)(1 \otimes B)$ throughout the paper. By (2.28) we have

$$
\begin{gathered}
\frac{1}{2 \pi i}\left(\left[R_{0}(\lambda+i 0)-R_{0}(\lambda-i 0)\right] f, f\right) \\
=\sinh (\sqrt{\lambda} \pi) \int_{\mathbf{R}^{n-1}}\left|\left(K(|\xi|) F_{0} f(\xi, \cdot)\right)(\sqrt{\lambda})\right|^{2} d \xi .
\end{gathered}
$$

With these preparations, we introduce the spectral representation for $H_{0}$.

Definition 3.2. We define

$$
\left(\mathcal{F}_{0}^{( \pm)} f\right)(w, \lambda)=\left(F_{0}^{-1}|\xi|^{\mp i \sqrt{\lambda}}\left(K(|\xi|)\left(F_{0} f\right)(\xi, \cdot)\right)(\sqrt{\lambda})\right)(w)
$$

Then by (3.11), we have

$$
\frac{1}{2 \pi i}\left(\left[R_{0}(\lambda+i 0)-R_{0}(\lambda-i 0)\right] f, f\right)=\sinh (\sqrt{\lambda} \pi) \int_{\mathbf{R}^{n-1}}\left|\left(\mathcal{F}_{0}^{( \pm)} f\right)(w, \lambda)\right|^{2} d w
$$

Using (2.29) and (2.30), one can show the following theorem. 


\section{Theorem 3.3.}

$$
\mathcal{F}_{0}^{( \pm)}: L^{2}\left(\mathbf{R}^{n} ; d x d y\right) \rightarrow L^{2}\left(\mathbf{R}^{n-1} \times(0, \infty) ; \sinh (\pi \sqrt{\lambda}) d w d \lambda\right)
$$

is unitary and diagonalizes $H_{0}$ :

$$
\left(\mathcal{F}_{0}^{( \pm)} H_{0} f\right)(w, \lambda)=\lambda\left(\mathcal{F}_{0}^{( \pm)} f\right)(w, \lambda)
$$

This spectral representation is related to the asymptotic behavior of the resolvent in the following way.

Theorem 3.4. For $f \in \mathcal{H}^{0, s}, s>1 / 2$, we have

$$
\begin{aligned}
\lim _{y \rightarrow-\infty} e^{ \pm i \sqrt{\lambda} y}\left(R_{0}(\lambda \pm i 0) f\right)(\cdot, y) & =C_{ \pm}(\lambda)\left(\mathcal{F}_{0}^{( \pm)} f\right)(\cdot, \lambda) \text { in } L^{2}\left(\mathbf{R}^{n-1}\right), \\
C_{ \pm}(\lambda) & =\frac{2^{ \pm i \sqrt{\lambda}} \pi}{\Gamma(1 \mp i \sqrt{\lambda})} .
\end{aligned}
$$

Proof. This follows from (2.18), (2.25) and Lebesgue's convergence theorem. $\diamond$

3.3 Spectral representation for $H$. One can construct the spectral representation for $H=H_{0}+V$ by the method of perturbation. We define for $\lambda>0$ and $f \in C_{0}^{\infty}\left(\mathbf{R}^{n}\right)$

$$
\left(\mathcal{F}_{0}^{( \pm)}(\lambda) f\right)(w)=\left(\mathcal{F}_{0}^{( \pm)} f\right)(w, \lambda) .
$$

By Theorem $3.1(4)$ and (3.12)

$$
\mathcal{F}_{0}^{( \pm)}(\lambda) \in \mathbf{B}\left(\mathcal{H}^{0, s} ; L^{2}\left(\mathbf{R}^{n-1}\right)\right), \quad s>1 / 2 .
$$

We define

$$
\mathcal{F}^{( \pm)}(\lambda)=\mathcal{F}_{0}^{( \pm)}(\lambda)(1-V R(\lambda \pm i 0)) .
$$

Then by Theorem 3.1 (3) and (3.14)

$$
\mathcal{F}^{( \pm)}(\lambda) \in \mathbf{B}\left(\mathcal{H}^{0, s} ; L^{2}\left(\mathbf{R}^{n-1}\right)\right), \quad s>1 / 2 .
$$

For $\varphi \in L^{2}\left(\mathbf{R}^{n-1}\right), \mathcal{F}^{( \pm)}(\lambda)^{*} \varphi \in \mathcal{H}^{0,-s}$ is a generalized eigenfunction in the following sense

$$
(H-\lambda) \mathcal{F}^{( \pm)}(\lambda)^{*} \varphi=0 .
$$

The following theorem is proved by the well-known method of perturbation. See e.g. Kuroda [20].

Theorem 3.5. Let $\left(\mathcal{F}^{( \pm)} f\right)(w, \lambda)=\left(\mathcal{F}^{( \pm)}(\lambda) f\right)(w)$. Then $\mathcal{F}^{( \pm)}$are uniquely extended to partial isometries with initial set $\mathcal{H}_{\text {ac }}(H)=$ the absolutely continuous subspace for $H$ and final set $L^{2}\left(\mathbf{R}^{n-1} \times(0, \infty) ; \sinh (\pi \sqrt{\lambda}) d w d \lambda\right)$. They diagonalize $H$ :

$$
\left(\mathcal{F}^{( \pm)} H f\right)(w, \lambda)=\lambda\left(\mathcal{F}^{( \pm)} f\right)(w, \lambda) .
$$

By the resolvent equation

$$
R(\lambda \pm i 0)=R_{0}(\lambda \pm i 0)(1-V R(\lambda \pm i 0))
$$

and Theorem 3.4, we have

Theorem 3.6. For $f \in \mathcal{H}^{0, s}, s>1 / 2$, we have

$$
\lim _{y \rightarrow-\infty} e^{ \pm i \sqrt{\lambda} y} R(\lambda \pm i 0) f(\cdot, y)=C_{ \pm}(\lambda) \mathcal{F}^{( \pm)}(\lambda) f \quad \text { in } \quad L^{2}\left(\mathbf{R}^{n-1}\right),
$$

where $C_{ \pm}(\lambda)$ are given in Theorem 3.4. 
3.4 Scattering amplitude. The wave operators $W_{ \pm}$are defined by

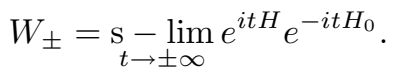

They are asymptotically complete in the sense that $\operatorname{Ran} W_{ \pm}=\mathcal{H}_{a c}(H)$. Let $S$ be the scattering operator

$$
S=W_{+}^{*} W_{-} .
$$

It is well-known that $\hat{S}=\mathcal{F}_{0}^{(+)} S \mathcal{F}_{0}^{(+) *}$ has the direct integral representation :

$$
(\hat{S} f)(w, \lambda)=(\hat{S}(\lambda) f(\cdot, \lambda))(w), \quad \forall f \in L^{2}\left(\mathbf{R}^{n-1} \times(0, \infty) ; \sinh (\pi \sqrt{\lambda}) d w d \lambda\right),(3.20)
$$

where $\hat{S}(\lambda)$ is untary on $L^{2}\left(\mathbf{R}^{n-1}\right)$ and is written as

$$
\begin{gathered}
\hat{S}(\lambda)=1-2 \pi i \sinh (\pi \sqrt{\lambda}) A(\lambda), \\
A(\lambda)=\mathcal{F}_{0}^{(+)}(\lambda)(V-V R(\lambda+i 0) V) \mathcal{F}_{0}^{(+)}(\lambda)^{*} .
\end{gathered}
$$

The last formula is called the scattering amplitude.

We next relate $A(\lambda)$ to the asymptotic behavior at infinity of the generalized eigenfunction of $H$. For $\lambda>0$, let $Q(\lambda)$ be defined by

$$
Q(\lambda)=F_{0}^{-1}|\xi|^{2 i \sqrt{\lambda}} F_{0}
$$

Then we have

$$
\mathcal{F}_{0}^{(-)}(\lambda)=Q(\lambda) \mathcal{F}_{0}^{(+)}(\lambda)
$$

With this in mind, we define the geometric scattering amplitude $\widetilde{A}(\lambda)$ by

$$
\widetilde{A}(\lambda)=A(\lambda) Q(\lambda)^{*} .
$$

Theorem 3.7. The geometric scattering amplitude $\widetilde{A}(\lambda)$ has the following expression

$$
\widetilde{A}(\lambda)=\mathcal{F}_{0}^{(+)}(\lambda)(V-V R(\lambda+i 0) V) \mathcal{F}_{0}^{(-)}(\lambda)^{*} .
$$

It is related to the asymptotic behavior of a generalized eigenfunction in the following way : For $\varphi \in L^{2}\left(\mathbf{R}^{n-1}\right)$, we have as $y \rightarrow-\infty$

$$
\mathcal{F}^{(-)}(\lambda)^{*} \varphi-\mathcal{F}_{0}^{(-)}(\lambda)^{*} \varphi \sim-C_{+}(\lambda) e^{-i \sqrt{\lambda} y} \widetilde{A}(\lambda) \varphi \quad \text { in } \quad L^{2}\left(\mathbf{R}^{n-1}\right) .
$$

Proof. The formula (3.26) follows from (3.22) and (3.24). The formula (3.27) follows from Theorem 3.6.

\section{SCHRÖDINGER OPERATORS ON $\Gamma \backslash \mathbf{H}^{n}$}

4.1 Floquet operators on the quotient manifold. Let $\Gamma \subset \mathbf{R}^{n-1}$ be a lattice of rank $n-1$, and $\Gamma^{*}=\left\{\gamma^{*} \in \mathbf{R}^{n-1} ; \gamma \cdot \gamma^{*} \in 2 \pi \mathbf{Z}, \forall \gamma \in \Gamma\right\}$ be its dual lattice. The fundamental domains of $\Gamma$ and $\Gamma^{*}$ are

$$
\mathbf{E}=\mathbf{R}^{n-1} / \Gamma, \quad \mathbf{E}^{*}=\mathbf{R}^{n-1} / \Gamma^{*} .
$$

By the natural identification of $\Gamma$ with the discrete translation group acting on $\mathbf{H}^{n}$, we introduce the hyperbolic manifold $\mathcal{M}=\Gamma \backslash \mathbf{H}^{n}$, whose Laplace-Beltrami operator is

$$
\Delta_{\mathcal{M}}=x_{n}^{2} \partial_{n}^{2}-(n-2) x_{n} \partial_{n}-x_{n}^{2} P_{0}
$$

where $P_{0}=-\Delta_{x}$ is the Laplacian on $\mathbf{E}$ with periodic boundary condition. As in $\S 3$, the change of variable $y=\log x_{n}$ transforms $-\Delta_{\mathcal{M}}-(n-1)^{2} / 4$ to

$$
H_{0}=-\partial_{y}^{2}+e^{2 y} P_{0} \quad \text { in } \quad L^{2}(\mathbf{E} \times \mathbf{R} ; d x d y) .
$$


For $\theta \in \mathbf{E}^{*}$, we introduce the following operator on $\mathbf{E} \times \mathbf{R}$

$$
H_{0}(\theta)=-\partial_{y}^{2}+e^{2 y} P_{0}(\theta), \quad P_{0}(\theta)=\left(-i \partial_{x}+\theta\right)^{2},
$$

where $\partial_{x}=\nabla_{x}$ and the periodic boundary condition is imposed on $P_{0}(\theta)$.

Let $H(\theta)=H_{0}(\theta)+V$. We assume that $V(x, y)$ is a real function and is split into two parts $V=V_{1}+V_{2}$, where $V_{1}(x, y)$ is compactly supported and

$$
V_{1}(x, y) \in L^{p}(\mathbf{E} \times \mathbf{R}),
$$

with $p=2(n \leq 3), p>n / 2(n \geq 4)$, and where $V_{2}$ verifies

$$
|V(x, y)| \leq C(1+|y|)^{-1-\epsilon}
$$

for some constants $C, \epsilon>0$.

$\left.H(\theta)\right|_{C_{0}^{\infty}(\Omega)}$ is essentially self-adjoint, whose self-adjoint extension is denoted by $H(\theta)$ again. By the reasoning to be given in 4.4, we call $H(\theta)$ Floquet operator. Let $R(z ; \theta)=(H(\theta)-z)^{-1}$, and $R_{0}(z ; \theta)=\left(H_{0}(\theta)-z\right)^{-1}$. For $s \in \mathbf{R}$, let $L^{2, s}$ be the weighted Hilbert space equipped with the norm

$$
\|u\|_{s}^{2}=\int_{\Omega}(1+|y|)^{2 s}|u(x, y)|^{2} d x d y<\infty .
$$

We shall prove the following theorem in $\S 8$.

Theorem 4.1. (1) $\sigma_{e}(H(\theta))=[0, \infty)$.

(2) $\sigma_{p}(H(\theta)) \cap(0, \infty)=\emptyset$.

(3) For $s>1 / 2$ and $\lambda>0$, there exist the strong limits

$$
\mathrm{s}-\lim _{\epsilon \downarrow 0} R(\lambda \pm i \epsilon ; \theta)=: R(\lambda \pm i 0 ; \theta) \in \mathbf{B}\left(L^{2, s} ; L^{2,-s}\right) .
$$

4.2 Spectral representation for $H_{0}(\theta)$. We put

$$
\hat{f}\left(\gamma^{*}, y\right)=\int_{\mathbf{E}} e^{-i \gamma^{*} \cdot x} f(x, y) d x .
$$

Then as in (3.10), we have for $f \in C_{0}^{\infty}(\Omega)$

$$
R_{0}(\lambda \pm i 0 ; \theta) f=\frac{1}{|\mathbf{E}|} \sum_{\gamma^{*} \in \Gamma^{*}} e^{i \gamma^{*} \cdot x}\left(L_{0}\left(\left|\gamma^{*}+\theta\right|\right)-\lambda \mp i 0\right)^{-1} \hat{f}\left(\gamma^{*}, \cdot\right)
$$

Definition 4.2. We define

$$
\left(\mathcal{F}_{0}^{( \pm)}(\theta) f\right)(w, \lambda)=\sum_{\gamma^{*} \in \Gamma^{*}} e^{i \gamma^{*} \cdot w}\left|\gamma^{*}+\theta\right|^{\mp i \sqrt{\lambda}}\left(K\left(\left|\gamma^{*}+\theta\right|\right) \hat{f}\left(\gamma^{*}, \cdot\right)\right)(\sqrt{\lambda}) \text {. }
$$

Then by (2.28) and (4.9), we have

$$
\frac{1}{2 \pi i}\left(\left[R_{0}(\lambda+i 0 ; \theta)-R_{0}(\lambda-i 0 ; \theta)\right] f, f\right)=\sinh (\sqrt{\lambda} \pi) \int_{\mathbf{E}}\left|\mathcal{F}_{0}^{( \pm)}(\theta) f(w, \lambda)\right|^{2} d w .
$$

One can show the following theorem as in $\S 3$.

Theorem 4.3. Assume $\theta \notin \Gamma^{*}$.

(1) $\mathcal{F}_{0}^{( \pm)}(\theta)$ is unitary

$$
\mathcal{F}_{0}^{( \pm)}(\theta): L^{2}(\Omega ; d x d y) \rightarrow L^{2}(\mathbf{E} \times(0, \infty) ; \sinh (\sqrt{\lambda} \pi) d w d \lambda)
$$

and diagonalizes $H_{0}(\theta)$ :

$$
\left(\mathcal{F}_{0}^{( \pm)}(\theta) H_{0}(\theta) f\right)(w, \lambda)=\lambda\left(\mathcal{F}_{0}^{( \pm)}(\theta) f\right)(w, \lambda) .
$$


(2) Let $f \in L^{2, s}, s>1 / 2$. Then we have

$$
\begin{gathered}
\lim _{y \rightarrow-\infty} e^{ \pm i \sqrt{\lambda} y}\left(R_{0}(\lambda \pm i 0 ; \theta) f\right)(\cdot, y)=C_{ \pm}(\lambda)\left(\mathcal{F}_{0}^{( \pm)}(\theta) f\right)(\cdot, \lambda) \text { in } L^{2}(\mathbf{E}), \\
C_{ \pm}(\lambda)=\frac{2^{ \pm i \sqrt{\lambda}} \pi}{\Gamma(1 \mp i \sqrt{\lambda})|\mathbf{E}|} .
\end{gathered}
$$

Proof. We give a sketch of the proof of (2). Using (2.18) we easily see

$$
\sup _{y} \|\left(R_{0}(\lambda+i 0 ; \theta) f(\cdot, y)\left\|_{L^{2}(\mathbf{E})} \leq C\right\| f \|_{s} .\right.
$$

To prove (2), therefore, we have only to prove it for $f \in C_{0}^{\infty}(\mathbf{E} \times \mathbf{R})$. Approximate $f$ by a finite sum of the Fourier series and apply (2.25).

4.3 Scattering amplitude. We define for $\lambda>0$ and $f \in C_{0}^{\infty}(\mathbf{E} \times \mathbf{R})$

$$
\left(\mathcal{F}_{0}^{( \pm)}(\lambda ; \theta) f\right)(w)=\left(\mathcal{F}_{0}^{( \pm)}(\theta) f\right)(w, \lambda) .
$$

Then by Theorem 4.1 and $(4.10)$

$$
\mathcal{F}_{0}^{( \pm)}(\lambda ; \theta) \in \mathbf{B}\left(L^{2, s} ; L^{2}(\mathbf{E})\right), \quad s>1 / 2 .
$$

We put

$$
\mathcal{F}^{( \pm)}(\lambda ; \theta)=\mathcal{F}_{0}^{( \pm)}(\lambda ; \theta)(1-V R(\lambda \pm i 0 ; \theta)) .
$$

By Theorem $4.3(2)$, we have the following theorem.

Theorem 4.4. Let $f \in L^{2, s}, s>1 / 2$. Then we have

$$
\begin{gathered}
\lim _{y \rightarrow-\infty} e^{ \pm i \sqrt{\lambda} y}(R(\lambda \pm i 0 ; \theta) f)(\cdot, y)=C_{ \pm}(\lambda) \mathcal{F}^{( \pm)}(\lambda ; \theta) f \text { in } L^{2}(\mathbf{E}), \\
C_{ \pm}(\lambda)=\frac{2^{ \pm i \sqrt{\lambda}} \pi}{\Gamma(1 \mp i \sqrt{\lambda})|\mathbf{E}|} .
\end{gathered}
$$

As in $\S 3$, we define (geometric) scattering amplitudes as follows :

$$
\begin{gathered}
A(\lambda ; \theta)=\mathcal{F}_{0}^{(+)}(\lambda ; \theta)(V-V R(\lambda+i 0 ; \theta) V) \mathcal{F}_{0}^{(+)}(\lambda ; \theta)^{*}, \\
\widetilde{A}(\lambda ; \theta)=A(\lambda ; \theta) Q(\lambda ; \theta)^{*}, \\
Q(\lambda ; \theta) \varphi=\frac{1}{|\mathbf{E}|} \sum_{\gamma^{*} \in \Gamma^{*}} e^{i \gamma^{*} \cdot x}\left|\gamma^{*}+\theta\right|^{2 i \sqrt{\lambda}} \hat{\varphi}\left(\gamma^{*}\right) .
\end{gathered}
$$

Since $\mathcal{F}_{0}^{(-)}(\lambda ; \theta)=Q(\lambda ; \theta) \mathcal{F}_{0}^{(+)}(\lambda ; \theta)$, the following theorem is proved in the same way as Theorem 3.7 .

Theorem 4.5. The geometric scattering amplitude $\widetilde{A}(\lambda ; \theta)$ has the following expression

$$
\widetilde{A}(\lambda ; \theta)=\mathcal{F}_{0}^{(+)}(\lambda ; \theta)(V-V R(\lambda+i 0 ; \theta) V) \mathcal{F}_{0}^{(-)}(\lambda ; \theta)^{*} .
$$

For $\varphi \in L^{2}(\mathbf{E})$, we have as $y \rightarrow-\infty$

$$
\begin{aligned}
\mathcal{F}^{(-)}(\lambda ; \theta)^{*} \varphi-\mathcal{F}_{0}^{(-)}(\lambda ; \theta)^{*} \varphi & -C_{+}(\lambda) e^{-i \sqrt{\lambda} y} \widetilde{A}(\lambda ; \theta) \varphi \text { in } L^{2}(\mathbf{E}) \\
C_{+}(\lambda) & =\frac{2^{i \sqrt{\lambda}} \pi}{\Gamma(1-i \sqrt{\lambda})|\mathbf{E}|} .
\end{aligned}
$$


4.4 Periodic Schrödinger operator. Suppose we are given a $\Gamma$-periodic Schrödinger operator $H_{0}+V$ on $\mathbf{H}^{n}$. A natural way to study its spectral structure is to investigate $H(\theta), \theta \in \mathbf{E}^{*}$, on $\Gamma \backslash \mathbf{H}^{n}$. In fact by the Floquet (or Bloch) theory it can be shown that (see e.g. [29] Vol 4, p. 279) there exists a unitary operator $U: L^{2}\left(\mathbf{H}^{n}\right) \rightarrow L^{2}\left(\mathbf{E} \times \mathbf{R} \times \mathbf{E}^{*}\right)$ such that

$$
\left(U\left(H_{0}+V\right) U^{-1} f\right)(x, y, \theta)=\left(\left(H_{0}(\theta)+V\right) f(\cdot, \theta)\right)(x, y)
$$

We do not use this property in this paper, however.

4.5 The case $\theta=0$. When $\theta=0$, the spectral representation of $H_{0}(0)$ should be modified at the mode $\gamma^{*}=0$. We put

$$
\begin{aligned}
& F_{0,+\infty}(\lambda) f=(4 \pi \sqrt{\lambda})^{-1 / 2} \int_{-\infty}^{\infty} e^{-i \sqrt{\lambda} y} \hat{f}(0, y) d y \\
& F_{0,-\infty}(\lambda) f=(4 \pi \sqrt{\lambda})^{-1 / 2} \int_{-\infty}^{\infty} e^{i \sqrt{\lambda} y} \hat{f}(0, y) d y
\end{aligned}
$$

Recall that $\hat{f}\left(\gamma^{*}, y\right)$ is defined by (4.8). We also put

$$
\mathcal{F}_{0}^{( \pm)}(\lambda ; 0) f(w)=\frac{1}{|\mathbf{E}|} \sum_{0 \neq \gamma^{*} \in \Gamma^{*}} e^{i \gamma^{*} \cdot w}\left|\gamma^{*}\right|^{\mp i \sqrt{\lambda}}\left(K\left(\left|\gamma^{*}\right|\right) \hat{f}\left(\gamma^{*}, \cdot\right)\right)(\sqrt{\lambda})
$$

Then we have

$$
\begin{aligned}
& \frac{1}{2 \pi i}\left(\left[R_{0}(\lambda+i 0 ; 0)-R_{0}(\lambda-i 0 ; 0)\right] f, f\right) \\
= & \left|F_{0,+\infty}(\lambda) f\right|^{2}+\left|F_{0,-\infty}(\lambda) f\right|^{2}+\sinh (\sqrt{\lambda} \pi)\left\|\mathcal{F}_{0}^{(+)}(\lambda ; 0) f\right\|_{L^{2}(\mathbf{E})}^{2}
\end{aligned}
$$

As in Theorem 4.3, the following theorem holds.

Theorem 4.6. (1) The mapping

$$
\begin{gathered}
L^{2}(\mathbf{E} \times \mathbf{R}, d x d y) \ni f \rightarrow\left(F_{0,+\infty}(\lambda) f, F_{0,-\infty}(\lambda) f, \mathcal{F}_{0}^{( \pm)}(\lambda ; 0) f\right) \\
\in \mathbf{C} \times \mathbf{C} \times L^{2}(\mathbf{E} \times(0, \infty) ; \sinh (\sqrt{\lambda} \pi) d w d \lambda)
\end{gathered}
$$

is unitary and diagonalizes $H_{0}$.

(1) Let $f \in L^{2, s}, s>1 / 2$. Then we have

$$
\begin{gathered}
\lim _{y \rightarrow-\infty} e^{ \pm i \sqrt{\lambda} y}\left(R_{0}(\lambda \pm i 0 ; 0) f\right)(\cdot, y)=C_{0}(\lambda) F_{0,-\infty}(\lambda) f+C_{ \pm}(\lambda) \mathcal{F}_{0}^{( \pm)}(\lambda ; 0) f \\
\lim _{y \rightarrow \infty} e^{\mp i \sqrt{\lambda} y}\left(R_{0}(\lambda \pm i 0 ; 0) f\right)(\cdot, y)=C_{0}(\lambda) F_{0,+\infty}(\lambda) f
\end{gathered}
$$

where $C_{0}(\lambda)=\pi^{1 / 2} \lambda^{-1 / 4}$ and $C_{ \pm}(\lambda)$ is from Theorem 4.3 (2).

As in (4.14), the spectral representation for $H$ is defined by

$$
\begin{gathered}
F_{+\infty}^{( \pm)}(\lambda)=F_{0,+\infty}(\lambda)(1-V R(\lambda \pm i 0 ; 0)), \\
F_{-\infty}^{( \pm)}(\lambda)=F_{0,-\infty}(\lambda)(1-V R(\lambda \pm i 0 ; 0)), \\
\mathcal{F}^{( \pm)}(\lambda ; 0)=\mathcal{F}_{0}^{( \pm)}(\lambda ; 0)(1-V R(\lambda \pm i 0 ; 0)) .
\end{gathered}
$$

Then the following theorem holds. 
Theorem 4.7. For $f \in L^{2, s}, s>1 / 2$, we have

$$
\begin{gathered}
\lim _{y \rightarrow-\infty} e^{ \pm i \sqrt{\lambda} y}(R(\lambda \pm i 0 ; 0) f)(\cdot, y)=C_{0}(\lambda) F_{-\infty}^{( \pm)}(\lambda) f+C_{ \pm}(\lambda) \mathcal{F}^{( \pm)}(\lambda ; 0) f, \\
\lim _{y \rightarrow \infty} e^{\mp i \sqrt{\lambda} y}(R(\lambda \pm i 0 ; 0) f)(\cdot, y)=C_{0}(\lambda) F_{+\infty}^{( \pm)}(\lambda) f
\end{gathered}
$$

$C_{0}(\lambda)$ and $C_{ \pm}(\lambda)$ being given in Theorem 4.6.

We finally define

$$
\begin{gathered}
\mathbf{F}_{0}^{( \pm)}(\lambda ; 0)=\left(F_{0,+\infty}(\lambda), F_{0,-\infty}(\lambda), \mathcal{F}_{0}^{( \pm)}(\lambda ; 0)\right), \\
\mathbf{F}^{( \pm)}(\lambda ; 0)=\left(F_{+\infty}^{( \pm)}(\lambda), F_{-\infty}^{( \pm)}(\lambda), \mathcal{F}^{( \pm)}(\lambda ; 0)\right),
\end{gathered}
$$

and define the (geometric) scattering amplitude as follows :

$$
\begin{aligned}
& \mathbf{A}(\lambda ; 0)=\mathbf{F}_{0}^{(+)}(\lambda ; 0)(V-V R(\lambda+i 0 ; 0) V) \mathbf{F}_{0}^{(+)}(\lambda ; 0)^{*}, \\
& \widetilde{\mathbf{A}}(\lambda ; 0)=\mathbf{F}_{0}^{(+)}(\lambda ; 0)(V-V R(\lambda+i 0 ; 0) V) \mathbf{F}_{0}^{(-)}(\lambda ; 0)^{*} .
\end{aligned}
$$

As in (4.16), $\widetilde{\mathbf{A}}(\lambda ; 0)$ is related with $\mathbf{A}(\lambda ; 0)$ in the following way :

$$
\begin{gathered}
\widetilde{\mathbf{A}}(\lambda ; 0)=\mathbf{A}(\lambda ; 0) \mathbf{Q}(\lambda ; 0)^{*}, \\
\mathbf{Q}(\lambda ; 0)=\left(\begin{array}{ccc}
1 & 0 & 0 \\
0 & 1 & 0 \\
0 & 0 & Q(\lambda ; 0)
\end{array}\right), \\
Q(\lambda ; 0) \varphi=\frac{1}{|\mathbf{E}|} \sum_{0 \neq \gamma^{*} \in \Gamma^{*}} e^{i \gamma^{*} \cdot x}\left|\gamma^{*}\right|^{2 i \sqrt{\lambda}} \hat{\varphi}\left(\gamma^{*}\right) .
\end{gathered}
$$

We also have the follwing theorem.

Theorem 4.8. For $\Phi \in \mathbf{C} \times \mathbf{C} \times L^{2}(\mathbf{E})$, we have as $y \rightarrow \infty$,

$$
\left(\mathbf{F}^{(-)}(\lambda ; 0)^{*} \Phi\right)_{1}-\left(\mathbf{F}_{0}^{(-)}(\lambda ; 0)^{*} \Phi\right)_{1} \sim C_{1}(\lambda) e^{i \sqrt{\lambda} y}(\widetilde{\mathbf{A}}(\lambda ; 0) \Phi)_{1},
$$

and as $y \rightarrow-\infty$

$$
\left(\mathbf{F}^{(-)}(\lambda ; 0)^{*} \Phi\right)_{2,3}-\left(\mathbf{F}_{0}^{(-)}(\lambda ; 0)^{*} \Phi\right)_{2,3} \sim C_{2,3}(\lambda) e^{-i \sqrt{\lambda} y}(\widetilde{\mathbf{A}}(\lambda ; 0) \Phi)_{2,3}
$$

Here for $\Psi=\left(a_{+}, a_{-}, \psi\right) \in \mathbf{C} \times \mathbf{C} \times L^{2}(\mathbf{E}),(\Psi)_{1}=a_{+}$and $(\Psi)_{2,3}=\left(a_{-}, \psi\right)$.

4.6 Scattering amplitude at the cusp. We shall observe the behavior of the resolvent as $y \rightarrow \infty$ and introduce the geometric scattering amplitude in the cusp neighborhood at infinity. In the sequel, the subscript $c$ means the cusp. We treat the case $\theta=0$.

We put for $\zeta>0$

$$
(I(\zeta) f)(\sigma)=\int_{-\infty}^{\infty} I_{i \sigma}\left(\zeta e^{y}\right) f(y) d y .
$$

Lemma 4.9. Let $s>1 / 2$ and $\sigma \in \mathbf{R}$. Then there exists a constant $C>0$ depending only on $s$ and $\sigma$ such that

$$
|(I(\zeta) f)(\sigma)| \leq C e^{\zeta^{2} / 2}\left(\int_{-\infty}^{\infty}\langle y\rangle^{2 s} \exp \left(e^{2 y}\right)|f(y)|^{2} d y\right)^{1 / 2}, \quad \forall \zeta>0 .
$$


Proof. By (2.5), we have $\left|I_{i \sigma}(z)\right| \leq C e^{z}, z>0$. Therefore using the inequality $\zeta e^{y} \leq\left(\zeta^{2}+e^{2 y}\right) / 2$, we get

$$
\begin{aligned}
|(I(\zeta) f)(\sigma)| & \leq C e^{\zeta^{2} / 2} \int_{-\infty}^{\infty} \exp \left(\frac{e^{2 y}}{2}\right)|f(y)| d y \\
& \leq C e^{\zeta^{2} / 2}\left(\int_{-\infty}^{\infty}\langle y\rangle^{2 s} \exp \left(e^{2 y}\right)|f(y)|^{2} d y\right)^{1 / 2} \cdot \diamond
\end{aligned}
$$

We use the notation

$$
\left\langle e^{i \gamma^{*} \cdot x}, f\right\rangle=\int_{\mathbf{E}} e^{i \gamma^{*} \cdot x} f(x, y) d x=\hat{f}\left(-\gamma^{*}, y\right) .
$$

Then by virtue of (2.31), we have as $y \rightarrow \infty$

$$
\left\langle e^{-i \gamma^{*} \cdot x}, R_{0}(\lambda \pm i 0 ; 0) f\right\rangle \sim \sqrt{\frac{\pi}{2\left|\gamma^{*}\right|}} \exp \left(-\frac{y}{2}-\left|\gamma^{*}\right| e^{y}\right)\left(I\left(\left|\gamma^{*}\right|\right) \hat{f}\left(\gamma^{*}, \cdot\right)\right)(\mp \sqrt{\lambda}),
$$

for $\gamma^{*} \neq 0$, and for $\gamma^{*}=0$

$$
\left\langle 1, R_{0}(\lambda \pm i 0 ; 0) f\right\rangle \sim \mp i \sqrt{\frac{\pi}{2 \lambda}} e^{ \pm i \sqrt{\lambda} y} F_{0, \pm \infty}(\lambda) \hat{f}(0, \cdot) .
$$

For $s \in \mathbf{R}$, let $L_{e x p}^{2, s}$ be the function space defined by

$$
L_{e x p}^{2, s} \ni f \Longleftrightarrow\|f\|_{s, \exp }=\left(\int_{\mathbf{E} \times \mathbf{R}}\langle y\rangle^{2 s} \exp \left(e^{2 y}\right)|f(x, y)|^{2} d x d y\right)^{1 / 2}<\infty .
$$

Lemma 4.9 implies that for $s>1 / 2$

$$
\left|\left(I\left(\left|\gamma^{*}\right|\right) \hat{f}\left(\gamma^{*}, \cdot\right)\right)(\mp \sqrt{\lambda})\right| \leq C e^{\left|\gamma^{*}\right|^{2} / 2}\|f\|_{s, \exp }
$$

with $C$ independent of $0 \neq \gamma^{*} \in \Gamma^{*}$.

In the following we shall assume that $V(x, y)$ is compactly supported. We define for $f \in L_{e x p}^{2, s}, s>1 / 2$,

$$
\begin{gathered}
\mathcal{F}_{0 c \gamma^{*}}^{( \pm)}(\lambda) f= \begin{cases}\left(I\left(\left|\gamma^{*}\right|\right) \hat{f}\left(\gamma^{*}, \cdot\right)\right)(\mp \sqrt{\lambda}), & \gamma^{*} \neq 0 \\
F_{0, \pm \infty}(\lambda) \hat{f}(0, \cdot), & \gamma^{*}=0\end{cases} \\
\mathcal{F}_{c \gamma^{*}}^{( \pm)}(\lambda)=\mathcal{F}_{0 c \gamma^{*}}^{( \pm)}(\lambda)(1-V R(\lambda \pm i 0 ; 0))
\end{gathered}
$$

We then have the following theorem.

Theorem 4.10. If $f \in L_{e x p}^{2, s}, s>1 / 2$, we have as $y \rightarrow \infty$

$$
\begin{gathered}
\left\langle e^{-i \gamma^{*} \cdot x}, R(\lambda \pm i 0 ; 0) f\right\rangle \sim \sqrt{\frac{\pi}{2\left|\gamma^{*}\right|}} \exp \left(-\frac{y}{2}-\left|\gamma^{*}\right| e^{y}\right) \mathcal{F}_{c \gamma^{*}}^{( \pm)}(\lambda) f, \quad \gamma^{*} \neq 0 \\
\langle 1, R(\lambda \pm i 0 ; 0) f\rangle \sim \mp i \sqrt{\frac{\pi}{2 \lambda}} e^{ \pm i \sqrt{\lambda} y} \mathcal{F}_{c \gamma^{*}}^{( \pm)}(\lambda) f, \quad \gamma^{*}=0 .
\end{gathered}
$$

We put

$$
\mu_{\gamma^{*}}=\left(1+\left|\gamma^{*}\right|\right)^{-n} e^{-\left|\gamma^{*}\right|^{2} / 2}
$$

and define on $L_{e x p}^{2, s}, s>1 / 2$,

$$
\begin{aligned}
& \mathcal{F}_{0 c}^{( \pm)}(\lambda)=\sum_{\gamma^{*} \in \Gamma^{*}} \mu_{\gamma^{*}} e^{i \gamma^{*} \cdot x} \mathcal{F}_{0 c \gamma^{*}}^{( \pm)}(\lambda), \\
& \mathcal{F}_{c}^{( \pm)}(\lambda)=\sum_{\gamma^{*} \in \Gamma^{*}} \mu_{\gamma^{*}} e^{i \gamma^{*} \cdot x} \mathcal{F}_{c \gamma^{*}}^{( \pm)}(\lambda) .
\end{aligned}
$$


By (4.39), we have

$$
\mathcal{F}_{0 c}^{( \pm)}(\lambda), \mathcal{F}_{c}^{( \pm)}(\lambda) \in \mathbf{B}\left(L_{\text {exp }}^{2, s} ; L^{2}(\mathbf{E})\right), \quad s>1 / 2 .
$$

The geometric scattering amplitude at the cusp is then defined by

$$
\widetilde{A}_{c}(\lambda)=\mathcal{F}_{0 c}^{(+)}(\lambda)(V-V R(\lambda+i 0 ; 0) V) \mathcal{F}_{0 c}^{(-)}(\lambda)^{*} .
$$

We also put

$$
\begin{aligned}
\widetilde{A}_{c \gamma^{*}}(\lambda) & =\left\langle e^{-i \gamma^{*} \cdot x}, \widetilde{A}_{c}(\lambda) \cdot\right\rangle \\
& =\mu_{\gamma^{*}} \mathcal{F}_{0 c \gamma^{*}}^{(+)}(\lambda)^{*}(V-V R(\lambda+i 0 ; 0) V) \mathcal{F}_{0 c}^{(-)}(\lambda)^{*}
\end{aligned}
$$

Note that for $\varphi \in L^{2}(\mathbf{E}), \mathcal{F}_{c}^{( \pm)}(\lambda)^{*} \varphi$ satisfies the Schrödinger equation $(H-\lambda) u=$ 0 , and it grows up at the cusp. In fact for $\varphi=e^{i \gamma^{*} \cdot x} / \gamma^{*}|\mathbf{E}|, \mathcal{F}_{0 c \gamma^{*}}^{( \pm)}(\lambda)^{*} \varphi=$ $I_{ \pm i \sqrt{\lambda}}\left(\left|\gamma^{*}\right| e^{y}\right)$, which behaves like $e^{z} / \sqrt{z}, z=\left|\gamma^{*}\right| e^{y}$, as $y \rightarrow \infty$. The following theorem, which follows easily from Theorem 4.10 , shows that $\widetilde{A}_{c}(\lambda)$ is obtained by observing this growing solution at the cusp.

Theorem 4.11. Let $\varphi \in L^{2}(\mathbf{E})$. For $\gamma^{*} \neq 0$, we have as $y \rightarrow \infty$

$$
\left\langle e^{-i \gamma^{*} \cdot x}, \mathcal{F}_{c}^{(-)}(\lambda)^{*} \varphi-\mathcal{F}_{0 c}^{(-)}(\lambda)^{*} \varphi\right\rangle \sim-\sqrt{\frac{\pi}{2\left|\gamma^{*}\right|}} \exp \left(-\frac{y}{2}-\left|\gamma^{*}\right| e^{y}\right) \widetilde{A}_{c \gamma^{*}}(\lambda) \varphi,
$$

For $\gamma^{*}=0$ we have as $y \rightarrow \infty$

$$
\left\langle 1, \mathcal{F}_{c}^{(-)}(\lambda)^{*} \varphi-\mathcal{F}_{0 c}^{(-)}(\lambda)^{*} \varphi\right\rangle \sim-i \sqrt{\frac{\pi}{2 \lambda}} e^{-i \sqrt{\lambda} y} \widetilde{A}_{c \gamma^{*}}(\lambda) \varphi
$$

\section{ReCOnstruction From SCATtering AmPlitudes}

We consider the inverse scattering problem on $\Gamma \backslash \mathbf{H}^{n}$. We use the same notation as in $\S 4$.

5.1 Analytic continuation of the resolvent. We take the fundamental domain $\mathbf{E}$ in such a way that it contains the origin in its interior. Let

$$
\Gamma_{\text {unit }}=\left\{\frac{\gamma}{|\gamma|} ; 0 \neq \gamma \in \Gamma\right\} .
$$

For $\alpha=\gamma /|\gamma| \in \Gamma_{\text {unit }}$ (given $\alpha$, we choose the smallest $\gamma$ ), we take $a, b$ such that

$$
-\frac{2 \pi}{|\gamma|}<-a<-b<0
$$

and put

$$
D_{\alpha}=\{z \in \mathbf{C} ;-a<\operatorname{Re} z<-b, \operatorname{Im} z>0\} .
$$

For $\gamma^{*} \in \Gamma^{*}$, we define

$$
\zeta\left(\gamma^{*}, z\right)=\sqrt{\left(\gamma^{*}+z \alpha\right)^{2}}
$$

Here we take the branch of $\sqrt{\cdot}$ such that $\operatorname{Re} \sqrt{\cdot} \geq 0$.

Lemma 5.1. $\zeta\left(\gamma^{*}, z\right)$ is analytic with respect to $z \in D_{\alpha}$ and there exists a constant $C>0$ such that

$$
\left|\zeta\left(\gamma^{*}, z\right)\right| \geq C \sqrt{\operatorname{Im} z}, \quad \forall \gamma^{*} \in \Gamma^{*}, \quad z \in D_{\alpha} .
$$


Proof. For $\gamma^{*} \in \Gamma^{*}$ such that $\gamma^{*} \cdot \alpha \neq 0,\left|\gamma^{*} \cdot \alpha\right| \geq 2 \pi /|\gamma|$. Therefore there exists $C>0$ such that

$$
\left|\operatorname{Im}\left(\gamma^{*}+z \alpha\right)^{2}\right| \geq C \operatorname{Im} z, \quad z \in D_{\alpha}
$$

This also holds for $\gamma^{*} \in \Gamma^{*}$ such that $\gamma^{*} \cdot \alpha=0$. This proves the lemma.

We put

$$
R_{0}(\lambda+i 0 ; z \alpha) f=\frac{1}{|\mathbf{E}|} \sum_{\gamma^{*} \in \Gamma^{*}} e^{i \gamma^{*} \cdot x} G\left(-\sqrt{\lambda}, \zeta\left(\gamma^{*}, z\right)\right) \hat{f}\left(\gamma^{*}, \cdot\right)
$$

where $G(\sigma, \lambda)$ is defined by $(2.20)$.

Fix $s>1 / 2$. We define the function spaces $X_{ \pm}$by

$$
u(x, y) \in X_{ \pm} \Longleftrightarrow\|u\|_{X_{ \pm}}^{2}=\int_{\mathbf{E} \times \mathbf{R}}(1+|y|)^{ \pm 2 s} e^{\mp y}|u(x, y)|^{2} d x d y<\infty .
$$

Lemma 5.2. (1) Let $s>1 / 2$. Then for $f \in L^{2, s}, R_{0}(\lambda+i 0 ; z \alpha) f$ is an $L^{2,-s}$ valued analytic finction of $z \in D_{\alpha}$ and as $z \rightarrow t \in(-a,-b), R_{0}(\lambda+i 0 ; z \alpha) f \rightarrow$ $R_{0}(\lambda+i 0 ; t \alpha) f$ in $L^{2,-s}$.

(2) There exists a constant $C>0$ such that

$$
\left\|R_{0}(\lambda+i 0 ; z \alpha) f\right\|_{-s} \leq C\|f\|_{s}, \quad \forall z \in D_{\alpha} .
$$

(3) For a constant $C>0$

$$
\left\|R_{0}(\lambda+i 0 ; z \alpha) f\right\|_{X_{-}} \leq \frac{C}{\sqrt{\operatorname{Im} z}}\|f\|_{X_{+}}, \quad \forall z \in D_{\alpha}
$$

Proof. For $f, g \in C_{0}^{\infty}(\Omega)$, we have

$$
\left(R_{0}(\lambda+i 0 ; z \alpha) f, g\right)=\frac{1}{|\mathbf{E}|} \sum_{\gamma^{*} \in \Gamma^{*}} \iint_{\mathbf{R}^{2}} G\left(y, y^{\prime} ;-\sqrt{\lambda}, \zeta\left(\gamma^{*}, z\right)\right) \hat{f}\left(\gamma^{*}, y^{\prime}\right) \overline{\hat{g}\left(\gamma^{*}, y\right)} d y^{\prime} d y
$$

Using (2.18), we have

$$
\begin{aligned}
\left|\left(R_{0}(\lambda+i 0 ; \theta) f, g\right)\right| & \leq C \iint \sum_{\gamma^{*} \in \Gamma^{*}}\left|\hat{f}\left(\gamma^{*}, y^{\prime}\right)\right| \cdot\left|\hat{g}\left(\gamma^{*}, y\right)\right| d y^{\prime} d y \\
& \leq C \int\left\|f\left(\cdot, y^{\prime}\right)\right\|_{L^{2}(\mathbf{E})} d y^{\prime} \int\|g(\cdot, y)\|_{L^{2}(\mathbf{E})} d y \\
& \leq C\|f\|_{s} \cdot\|g\|_{s},
\end{aligned}
$$

which proves (2). The assertion (1) follows from Lemma 5.1.

We also have by using (2.19), (5.7) and Lemma 5.1

$$
\begin{aligned}
\left|\left(R_{0}(\lambda+i 0 ; z \alpha) f, g\right)\right| & \leq \frac{C}{\sqrt{\operatorname{Im} z}} \int\left\|f\left(\cdot, y^{\prime}\right)\right\|_{L^{2}(\mathbf{E})} e^{-y^{\prime} / 2} d y^{\prime} \int\|g(\cdot, y)\|_{L^{2}(\mathbf{E})} e^{-y / 2} d y \\
& \leq \frac{C}{\sqrt{\operatorname{Im} z}}\|f\|_{X_{+}} \cdot\|g\|_{X_{+}},
\end{aligned}
$$

which implies (3).

We turn to the analytic continuation of $R(\lambda+i 0 ; t \alpha)$ with respect to $t$. We first consider the case $n \geq 3$. We assume that

$$
|V(x, y)| \leq C e^{-|y|}(1+|y|)^{-\rho}
$$


for some $\rho>1$. We take $1 / 2<s<\rho / 2$ and define the spaces $Y_{ \pm}$by

$$
u(x, y) \in Y_{ \pm} \Longleftrightarrow\|u\|_{Y_{ \pm}}^{2}=\int_{\mathbf{E} \times \mathbf{R}}(1+|y|)^{ \pm 2 s} e^{ \pm|y|}|u(x, y)|^{2} d x d y<\infty .
$$

Lemma 5.3. Let $n \geq 3$ and assume (5.8).

(1) $V R_{0}(\lambda+i 0 ; z \alpha)$ is a $\mathbf{B}\left(Y_{+} ; Y_{+}\right)$-valued analytic function of $z \in D_{\alpha}$.

(2) For each $z \in D_{\alpha}, V R_{0}(\lambda+i 0 ; z \alpha)$ is compact on $Y_{+}$.

(3) There exists a constant $C_{0}>0$ such that

$$
-1 \notin \sigma\left(V R_{0}(\lambda+i 0 ; z \alpha)\right) \text { if } \operatorname{Im} z>C_{0}, z \in D_{\alpha} .
$$

(4) $R(\lambda+i 0 ; z \alpha)$ defined by

$$
R(\lambda+i 0 ; z \alpha)=R_{0}(\lambda+i 0 ; z \alpha)\left(1+V R_{0}(\lambda+i 0 ; z \alpha)\right)^{-1}
$$

is a $\mathbf{B}\left(Y_{+} ; Y_{-}\right)$-valued meromorphic function of $z \in D_{\alpha}$. There exists a set of measure $0, \mathcal{E}$, in $(-a,-b)$ such that as $z \rightarrow t \in(-a,-b) \backslash \mathcal{E}, R(\lambda+i 0 ; z \alpha)$ has a boundary value, which coincides with $R(\lambda+i 0 ; t \alpha)=(H(t \alpha)-\lambda-i 0)^{-1}$.

(5) There exists a constant $C>0$ such that

$$
\|R(\lambda+i 0 ; z \alpha) f\|_{Y_{-}} \leq \frac{C}{\sqrt{\operatorname{Im} z}}\|f\|_{Y_{+}}, \quad z \in D_{\alpha}, \quad \operatorname{Im} z>C .
$$

Proof. The first three assertions follow from Lemma 5.2. To prove (2), note that $R_{0}(\lambda-i 0 ; \bar{z} \alpha) V$ is compact on $Y_{-}$. By the following Lemma $5.4, R(\lambda+i 0 ; z \alpha)$ is meromorphic in $D_{\alpha}$ and $R(\lambda+i 0 ; z \alpha)$ tends to some $S(t)$ as $z \rightarrow t \in(-a,-b) \backslash \mathcal{E}$, $\mathcal{E}$ being a null set. Then

$$
S(t)^{*}=R_{0}(\lambda-i 0 ; t \alpha)-R_{0}(\lambda-i 0 ; t \alpha) V S(t)^{*} .
$$

As will be explained in $\S 8$, for $g \in L^{2, s}, u=R_{0}(\lambda-i 0 ; \theta) g$ is an incoming solution of $\left(H_{0}(\theta)-\lambda\right) u=g$. Namely

$$
u \in L^{2,-s}, \quad F( \pm y>0)\left(i \partial_{y} \mp \sqrt{\lambda}\right) u \in L^{2,-\alpha}
$$

for some $0<\alpha<1 / 2<s$. Therefore, for $f \in Y_{+}, S(t)^{*} f$ is an incoming solution of the equation $\left(H_{0}(t \alpha)+V-\lambda\right) u=f$. Moreover, $R(\lambda-i 0 ; t \alpha) f$ is also an incoming solution and the incoming solution is unique. Therefore, $S(t)^{*} f=R(\lambda-i 0 ; t \alpha) f$, hence $S(t)=R(\lambda+i 0 ; t \alpha)$.

The assertion (5) follows from Lemma $5.2(3)$.

Lemma 5.4. Let $\mathcal{H}$ be a Hilbert space. Let $D$ be a unit disc in $\mathbf{C}$ and $f(z)$ a $\mathbf{B}(\mathcal{H})$-valued analytic function of $z \in D$, which is continuous on $\bar{D}$. Assume that $f(z)$ is compact for each $z \in \bar{D}$. Let $\mathcal{E}=\{z \in \bar{D} ; 1 \in \sigma(f(z))\}$, and suppose that $\mathcal{E} \neq \bar{D}$. Then $\mathcal{E} \cap D$ is discrete, $\mathcal{E} \cap \partial D$ is a closed 1-dimensional null set and for $z_{0} \in \partial D \backslash \mathcal{E},(1-f(z))^{-1} \rightarrow\left(1-f\left(z_{0}\right)\right)^{-1}$ as $D \backslash \mathcal{E} \ni z \rightarrow z_{0}$.

This lemma follows from the analytic Fredholm theorem (see e.g. [29] Vol. 1, p. 201) and the well-known Fatou-Riesz theorem on the boundary value of analytic functions (see e.g. [38] p. 135).

5.2 Estimates in 2-dimension. When $n=2$, one can allow $L^{2}$-local singularities for the potential. 
Lemma 5.5. Let $n=2$ and assume that

$$
e^{-y / 2} V(x, y) \in L^{2}(\mathbf{E} \times \mathbf{R})
$$

Then we have for $z \in D_{\alpha}$ and $s>1 / 2$

$$
\left\|V R_{0}(\lambda+i 0 ; z \alpha) f\right\|_{L^{2}(\mathbf{E} \times \mathbf{R})} \leq \frac{C}{\sqrt{\operatorname{Im} z}}\left\|e^{-y / 2} V\right\|_{L^{2}(\mathbf{E} \times \mathbf{R})}\left\|\langle y\rangle^{s} e^{-y / 2} f\right\|_{L^{2}(\mathbf{E} \times \mathbf{R})} .
$$

Proof. One can assume without loss of generality that $\Gamma^{*}=\mathbf{Z}$. Letting $\operatorname{Im} z=\tau$, we have

$$
|\zeta(n, z)| \geq C(|n|+\tau)
$$

for a constant $C>0$. Then $u(x, y)=R_{0}(\lambda+i 0 ; z \alpha) f$ is written as

$$
\begin{gathered}
u(x, y)=\sum_{n} a(n, y) e^{i n x} \\
|a(n, y)| \leq \frac{C}{|n|+\tau} e^{-y / 2} \int_{-\infty}^{\infty} e^{-y^{\prime} / 2}\left|\hat{f}\left(n, y^{\prime}\right)\right| d y^{\prime}
\end{gathered}
$$

By the Schwarz and the Parseval (in)equalities

$$
|u(x, y)|^{2} \leq C\left(\sum_{n \in \mathbf{Z}}(|n|+\tau)^{-2}\right) e^{-y}\left(\int_{\mathbf{E} \times \mathbf{R}}\left\langle y^{\prime}\right\rangle^{2 s} e^{-y^{\prime}}\left|f\left(x, y^{\prime}\right)\right|^{2} d x d y^{\prime}\right),
$$

which implies the lemma.

Lemma 5.6. When $n=2$, Lemma 5.3 holds under the assumption that $V=$ $V_{1}+V_{2}$, where $V_{1} \in L^{2}(\mathbf{E} \times \mathbf{R})$ and is compactly supported, $V_{2}$ satisfies (5.8).

Proof. Pick $\chi \in C_{0}^{\infty}(\mathbf{E} \times \mathbf{R})$ such that $\chi=1$ on supp $V_{1}$. Making use of the formula

$$
V_{1} R_{0}(\lambda+i 0 ; z \alpha)=V_{1}(-\Delta+1)^{-1}(-\Delta+1) \chi R_{0}(\lambda+i 0 ; z \alpha),
$$

one can see that $\left\|V_{1} R_{0}(\lambda+i 0 ; z \alpha)\right\|_{\mathbf{B}\left(Y_{+} ; Y_{+}\right)}$is locally bounded on $\overline{D_{\alpha}}$. For $f, g \in$ $C_{0}^{\infty}(\mathbf{E} \times \mathbf{R}),\left(R_{0}(\lambda+i 0 ; z \alpha) f, V g\right)$ is analytic with respect to $z \in D_{\alpha}$. This proves the analyticity of $V R_{0}(\lambda+i 0 ; z \alpha)$ in $\mathbf{B}\left(Y_{+} ; Y_{+}\right)$.

We split $V_{1}=V_{1, \epsilon}+V_{\infty, \epsilon}$ in such a way that $\left\|V_{1, \epsilon}\right\|_{L^{2}}<\epsilon, V_{\infty, \epsilon} \in L^{\infty}$. Then $\left\|R_{0}(\lambda-i 0 ; \bar{z} \alpha) V_{1, \epsilon}\right\|_{\mathbf{B}\left(Y_{-} ; Y_{-}\right)} \leq C \epsilon$ and $R_{0}(\lambda-i 0 ; \bar{z} \alpha) V_{\infty, \epsilon}$ is compact. Therefore $V R_{0}(\lambda+i 0 ; z \alpha)$ is compact on $Y_{+}$.

The assertion (4) is proven without any change, and the assertions (3) and (5) follow from Lemma 5.5.

5.3 Reconstruction of the potential. We are now in a position of extracting Fourier coefficients of $V(x, y)$ from scattering amplitudes $A(\lambda ; \theta), \theta \in \mathbf{E}^{*}$. The following assumption is imposed on $V$ :

(A) If $n \geq 3$, there exist $\rho>1$ and $\epsilon>0$ such that

$$
|V(x, y)| \leq \begin{cases}C \exp \left(-\epsilon e^{y}\right) & (y>0) \\ C\langle y\rangle^{-\rho} e^{y} & (y<0)\end{cases}
$$


If $n=2, V=V_{1}+V_{2}$, where $V_{1} \in L^{2}(\mathbf{E} \times \mathbf{R})$ and is compactly supported, and $V_{2}$ satisfies

$$
\left|V_{2}(x, y)\right| \leq \begin{cases}C_{N} \exp \left(-N e^{y}\right) & (y>0) \\ C\langle y\rangle^{-\rho} e^{y} & (y<0) .\end{cases}
$$

for a fixed $\rho>1$ and any $N>1$.

By Definition 4.2 and (2.3)

$$
\mathcal{F}_{0}^{(+)}(\lambda ; \theta)^{*} \varphi=\frac{1}{\pi} \sum_{\gamma^{*} \in \Gamma^{*}} e^{i \gamma^{*} \cdot x}\left|\gamma^{*}+\theta\right|^{i \sqrt{\lambda}} K_{i \sqrt{\lambda}}\left(\left|\gamma^{*}+\theta\right| e^{y}\right) \int_{\mathbf{E}} e^{-i \gamma^{*} \cdot w} \varphi(w) d w
$$

Therefore for $\xi \in \Gamma^{*}$

$$
\mathcal{F}_{0}^{(+)}(\lambda ; \theta)^{*} e^{i \xi \cdot w}=\frac{|\mathbf{E}|}{\pi} e^{i \xi \cdot x}|\xi+\theta|^{i \sqrt{\lambda}} K_{i \sqrt{\lambda}}\left(|\xi+\theta| e^{y}\right) .
$$

By making use of (2.3) and (5.15), we have

$$
\begin{aligned}
\left(A(\lambda ; \theta) e^{i \xi \cdot w}, e^{i \eta \cdot w}\right)= & \left(\frac{|\mathbf{E}|}{\pi}\right)^{2}\left(\frac{|\xi+\theta|}{|\eta+\theta|}\right)^{i \sqrt{\lambda}} \\
\times & \iint(V-V R(\lambda+i 0 ; \theta) V)\left[e^{i \xi \cdot x} K_{i \sqrt{\lambda}}\left(|\xi+\theta| e^{y}\right)\right] . \\
& e^{-i \eta \cdot x} K_{i \sqrt{\lambda}}\left(|\eta+\theta| e^{y}\right) d x d y .
\end{aligned}
$$

Let $k \in \Gamma^{*} \backslash\{0\}$ be arbitrarily given. If $n \geq 3$, a simple trigonometry shows that one can pick $\xi, \eta \in \Gamma^{*}$ and $\alpha \in \Gamma_{\text {unit }}$ such that

$$
k=\eta-\xi, \quad \alpha \cdot \eta<\delta<\alpha \cdot \xi<\epsilon
$$

for small $\delta>0$, where $\epsilon$ is the constant specified in the assumption (A). If $n=2$, by taking $\eta=0, \xi=-k$ and $\alpha=-k /|k|=-\operatorname{sgn} k$, one has

$$
k=\eta-\xi, \quad \alpha \cdot \eta<\delta<\alpha \cdot \xi
$$

for small $\delta>0$. We put $z=-\delta+i \tau$. Then since $\alpha \cdot \xi-\delta>0, \alpha \cdot \eta-\delta<0$, we have as $\tau \rightarrow \infty$,

$$
\begin{gathered}
\sqrt{(\xi+z \alpha)^{2}} \sim \tau i+(\alpha \cdot \xi-\delta), \\
\sqrt{(\eta+z \alpha)^{2}} \sim-\tau i-(\alpha \cdot \eta-\delta) .
\end{gathered}
$$

Therefore we have by $(2.7)$

$$
\begin{aligned}
K_{i \sqrt{\lambda}}\left(\sqrt{(\xi+z \alpha)^{2}} e^{y}\right) & \sim \frac{C}{\sqrt{\tau e^{y}}} \exp \left(-i \tau e^{y}-(\alpha \cdot \xi-\delta) e^{y}\right), \\
K_{i \sqrt{\lambda}}\left(\sqrt{(\eta+z \alpha)^{2}} e^{y}\right) & \sim \frac{C}{\sqrt{\tau e^{y}}} \exp \left(i \tau e^{y}+(\alpha \cdot \eta-\delta) e^{y}\right) .
\end{aligned}
$$

Now for $\epsilon$ small enough, we consider $\left(A(\lambda ; t \alpha) e^{i \xi \cdot w}, e^{i \eta \cdot w}\right)$ for $t \in(-2 \epsilon,-\epsilon)$. By virtue of (4.15), Lemma 5.3 and the well-known Riesz's theorem on boundary values of analytic functions, it has a unique meromorphic extension to $D_{\alpha}$. Let $z=-\delta+i \tau$, where $\epsilon<\delta<2 \epsilon$, and put

$$
B(\tau, \alpha, \xi, \eta)=\tau\left(A(\lambda ; z \alpha) e^{i \xi \cdot w}, e^{i \eta \cdot w}\right) .
$$


We let $\tau \rightarrow \infty$. By the assumption (A), the term containing $R(\lambda+i 0 ; z \alpha)$ disappears by virtue of Lemma $5.3(5)$. Hence we have by (5.20) and (5.21)

$$
\begin{aligned}
\lim _{\tau \rightarrow \infty} B(\tau, \alpha, \xi, \eta) & =\lim _{\tau \rightarrow \infty} \tau\left(V \mathcal{F}_{0}^{(+)}(\lambda ; \theta)^{*} e^{i \xi \cdot w}, \mathcal{F}_{0}^{(+)}(\lambda ; \theta)^{*} e^{i \eta \cdot w}\right) \\
& =\text { Const. } \int \hat{V}(k, y) e^{-y} \exp \left(\alpha \cdot k e^{y}\right) d y
\end{aligned}
$$

If $n \geq 3$, one can vary $\alpha$ as long as it satisfies (5.16). Let us take notice of the fact that the set $\Gamma_{\text {unit }}$ is dense in $S^{n-2}$. Then by the analytic continuation, one can reconstruct

$$
\int_{\mathbf{E} \times(0, \infty)} e^{-i k \cdot x} e^{-i t z} V(x, \log z) \frac{d x d z}{z^{2}}, \quad k \neq 0
$$

from the scattering amplitudes $\{A(\lambda ; \theta)\}_{\theta \in \mathbf{E}^{*}}$. If $n=2, \alpha \cdot k=-|k|$. Therefore we can reconstruct

$$
\int_{\mathbf{E} \times(0, \infty)} e^{-i k \cdot x} e^{-|k| z} V(x, \log z) \frac{d x d z}{z^{2}}, \quad k \neq 0
$$

from the scattering amplitudes $\{A(\lambda ; \theta)\}_{\theta \in \mathbf{E}^{*}}$. We have thus proven

Theorem 5.7. (ISP in $\left.\mathbf{H}^{n} / \Gamma\right)$. Assume $(A)$.

(1) If $n \geq 3$, one can uniquely reconstruct

$$
\int_{\mathbf{E}} e^{-i k \cdot x} V(x, y) d x, \quad \forall k \in \Gamma^{*} \backslash\{0\}
$$

from the scattering amplitudes $\{A(\lambda ; \theta)\}_{\theta \in \mathbf{E}^{*}}$ of arbitrarily fixed energy $\lambda>0$.

(2) If $n=2$, one can uniquely reconstruct

$$
\int_{\mathbf{E} \times(0, \infty)} e^{-i k \cdot x} e^{-|k| z} V(x, \log z) \frac{d x d z}{z^{2}}, \quad \forall k \in \Gamma^{*} \backslash\{0\}
$$

from the scattering amplitudes $\{A(\lambda ; \theta)\}_{\theta \in \mathbf{E}^{*}}$ of arbitrarily fixed energy $\lambda>0$.

\section{INVERSE BOUNDARY VALUE PROBLEM}

6.1 IBVP in $\Gamma \backslash \mathbf{H}^{n}$. Let $U$ be a bounded domain in $\mathbf{E} \times \mathbf{R}$ with smooth boundary $S$. For $q(x, y)$, real-valued, we consider the following boundary value problem

$$
\left(H_{0}(\theta)+q\right) u=0 \quad \text { in } \quad U,\left.\quad u\right|_{S}=f \in H^{3 / 2}(S) .
$$

Let $H_{0}(\theta)_{D}$ be $-e^{2 y}\left(\partial_{x}+i \theta\right)^{2}-\partial_{y}^{2}$ with Dirichlet boundary condition. For the solution $u$ of $(6.1)$, the D-N map is defined by

$$
\Lambda_{q}(\theta) f=\left.\left(e^{2 y} \nu_{x} \cdot\left(\partial_{x}+i \theta\right) u+\nu_{y} \partial_{y} u\right)\right|_{S},
$$

where $\nu_{e}=\left(\nu_{s}, \nu_{y}\right)$ is the outer unit normal to $S$ with respect to the Euclidean metric $(d x)^{2}+(d y)^{2}$.

Lemma 6.1. Let $U$ be a bounded domain in $\mathbf{E} \times \mathbf{R}$ with smooth boundary such that $(\mathbf{E} \times \mathbf{R}) \backslash \bar{U}$ is connected. Suppose $n \geq 3$ and that $q \in L^{\infty}(U)$. If $0 \notin \sigma\left(H_{0}(\theta)_{D}+q\right)$ for all $\theta \in \mathbf{E}^{*}$, then one can uniquely reconstruct

$$
\int_{\mathbf{E}} e^{-i k \cdot x} q(x, y) d x, \quad \forall k \in \Gamma^{*} \backslash\{0\}
$$

from $\Lambda_{q}(\theta)$ of all $\theta \in \mathbf{E}^{*}$. 
Admitting this lemma for the moment, we consider its applications.

6.2 IBVP in $\mathbf{H}^{n}$. Let $U$ be a bounded domain in $\mathbf{R}^{n-1} \times \mathbf{R}$ with smooth boundary $S$. Let $H_{0}=-e^{2 y} \Delta_{x}-\partial_{y}^{2}$. For a real-valued function $q(x, y)$, we study

$$
\left(H_{0}+q\right) u=0 \quad \text { in } \quad U,\left.\quad u\right|_{S}=f \in H^{3 / 2}(S) .
$$

Let $H_{0 D}$ be $-e^{2 y} \Delta_{x}-\partial_{y}^{2}$ with Dirichlet boundary condition. For the solution $u$ of (6.3), we define the D-N map $\Lambda_{q}$ by

$$
\Lambda_{q} f=\left.\left(e^{2 y} \nu_{x} \cdot \partial_{x} u+\nu_{y} \partial_{y} u\right)\right|_{S},
$$

where $\nu_{e}=\left(\nu_{x}, \nu_{y}\right)$ is the outer unit normal to $S$ with respect to the Euclidean metric $(d x)^{2}+(d y)^{2}$.

Lemma 6.2. Let $U$ be a bounded domain in $\mathbf{R}^{n-1} \times \mathbf{R}$ with smooth boundary such that $\left(\mathbf{R}^{n-1} \times \mathbf{R}\right) \backslash \bar{U}$ is connected. Suppose $n \geq 3$ and that $q \in L^{\infty}(U)$. If $0 \notin \sigma\left(H_{0 D}+q\right)$, then

$$
\int_{\mathbf{R}^{n-1}} e^{-k \cdot x} V(x, y) d x, \quad \forall k \in\left(\frac{2 \pi}{L} \mathbf{Z}\right)^{n-1} \backslash\{0\}
$$

is uniquely reconstructed from $\Lambda_{q}$, where $L>0$ is a constant such that $U \subset$ $[L / 3,2 L / 3]^{n-1} \times \mathbf{R}$.

Proof. Take the lattice $\Gamma \subset \mathbf{R}^{n-1}$ such that $\mathbf{R}^{n-1} / \Gamma=[0, L]^{n-1}$. We regard $[0, L]^{n-1}$ as a torus. However $U$ is contained in one coordinate patch of $[0, L]^{n-1} \times \mathbf{R}$. Then since $e^{-i x \cdot \theta} H_{0 D} e^{i x \cdot \theta}=H_{0}(\theta)_{D}$, we have

$$
e^{-i x \cdot \theta} \Lambda_{q} e^{i x \cdot \theta}=\Lambda_{q}(\theta) .
$$

Therefore, one can construct $\Lambda_{q}(\theta)$ from $\Lambda_{q}$. Lemma 6.2 then follows from Lemma 6.1 .

6.3 IBVP in $\mathbf{R}^{n}$. Let $\widetilde{U}$ be a bounded domain in the Euclidean space $\mathbf{R}^{n}$ with smooth boundary $\widetilde{S}$. For $V\left(x, x_{n}\right)$, real-valued, we study the boundary value problem

$$
(-\Delta+V) u=0 \quad \text { in } \quad \widetilde{U},\left.\quad u\right|_{\tilde{S}}=f \in H^{3 / 2}(\widetilde{S}) .
$$

Let $\Delta_{D}$ be $\Delta=\partial_{x}^{2}+\partial_{n}^{2}$ with Dirichlet boundary condition. The D-N map is defined by

$$
\widetilde{\Lambda}_{V} f=\left.\left(\widetilde{\nu}_{x} \cdot \partial_{x} u+\widetilde{\nu}_{n} \partial_{n} u\right)\right|_{\widetilde{S}}
$$

for the solution $u$ to (6.6). Here $\widetilde{\nu}_{e}=\left(\widetilde{\nu}_{x}, \widetilde{\nu}_{n}\right)$ is the outer unit normal to $\widetilde{S}$ with respect to the Euclidean metric $(d x)^{2}+\left(d x_{n}\right)^{2}$ and $\partial_{n}=\partial / \partial x_{n}$.

As is stated in the introduction, the following theorem was proved by SylvesterUhlmann [36], Nachman [25] and Khenkin-Novikov [19], and the results has been extended to various cases. However they were proved by essentially the same machinery, complex geometrical optics solution or Faddeev's Green function. We shall give here an alternative proof by using hyperbolic manifolds as a tool.

Theorem 6.3. (IBVP in $\mathbf{R}^{n}$ ). Let $\widetilde{U}$ be a bounded domain in $\mathbf{R}^{n}$ with smooth boundary such that $\mathbf{R}^{n} \backslash \overline{\widetilde{U}}$ is connected. Suppose $n \geq 3$ and that $V \in L^{\infty}(\widetilde{U})$. If $0 \notin \sigma\left(-\Delta_{D}+V\right)$, then $V\left(x, x_{n}\right)$ is uniquely reconstructed from $\widetilde{\Lambda}_{V}$. 
Proof. One can assume that $\widetilde{U} \subset\left\{\left(x, x_{n}\right) \in \mathbf{R}^{n} ; x_{n}>1\right\}$. Let $y=\log x_{n}$ and $u=e^{-y / 2} v$. Then if $u$ satisfies (6.6), $v$ satisfis

$$
\left(-e^{2 y} \partial_{x}^{2}-\partial_{y}^{2}\right) v+\left(e^{2 y} V+\frac{1}{4}\right) v=0 \quad \text { in } \quad U,\left.\quad v\right|_{S}=e^{y / 2} f \in H^{3 / 2}(S),
$$

where $U$ and $S$ are obtained from $\widetilde{U}$ and $\widetilde{S}$ by the change of variable. We show that

$$
\left.\left(e^{2 y} \nu_{x} \cdot \partial_{x}+\nu_{y} \partial_{y}\right)\right|_{S}=\left.\lambda\left(x, x_{n}\right) x_{n}^{2}\left(\widetilde{\nu}_{x} \cdot \partial_{x}+\widetilde{\nu}_{n} \partial_{n}\right)\right|_{\widetilde{S}}
$$

for a smooth function $\lambda\left(x, x_{n}\right)>0$ on $\widetilde{S}$. Here $\nu_{e}=\left(\nu_{x}, \nu_{y}\right)$ and $\widetilde{\nu}_{e}=\left(\widetilde{\nu}_{x}, \widetilde{\nu}_{n}\right)$ are the outer unit normals to $S$ and $\widetilde{S}$ with respect to the Euclidean metrics $(d x)^{2}+(d y)^{2}$ and $(d x)^{2}+\left(d x_{n}\right)^{2}$.

Suppose that $S$ is locally represented as $\{(x, y) ; \varphi(x, y)=0\}$ and put $\widetilde{\varphi}\left(x, x_{n}\right)=$ $\varphi\left(x, \log x_{n}\right)$. Then

$$
\begin{array}{ll}
\nu_{e}=c\left(\partial_{x} \varphi, \partial_{y} \varphi\right), & c=\left(\left|\partial_{x} \varphi\right|^{2}+\left|\partial_{y} \varphi\right|^{2}\right)^{-1 / 2} \\
\widetilde{\nu}_{e}=\widetilde{c}\left(\partial_{x} \widetilde{\varphi}, \partial_{n} \widetilde{\varphi}\right), & \widetilde{c}=\left(\left|\partial_{x} \widetilde{\varphi}\right|^{2}+\left|\partial_{n} \widetilde{\varphi}\right|^{2}\right)^{-1 / 2}
\end{array}
$$

Then we have

$$
\begin{gathered}
\frac{\widetilde{\nu}_{x}}{\widetilde{c}}=\partial_{x} \widetilde{\varphi}=\partial_{x} \varphi=\frac{\nu_{x}}{c}, \\
\frac{\widetilde{\nu}_{n}}{\widetilde{c}}=\partial_{n} \widetilde{\varphi}=\frac{1}{x_{n}} \partial_{y} \varphi=\frac{\nu_{y}}{x_{n} c} .
\end{gathered}
$$

By a simple computation, one can show $(6.9)$ with $\lambda\left(x, x_{n}\right)$ replaced by

$$
\frac{c}{\widetilde{c}}=\left(\frac{\left|\partial_{x} \widetilde{\varphi}\right|^{2}+x_{n}^{2}\left|\partial_{n} \widetilde{\varphi}\right|^{2}}{\left|\partial_{x} \widetilde{\varphi}\right|^{2}+\left|\partial_{n} \widetilde{\varphi}\right|^{2}}\right)^{-1 / 2} .
$$

Note that $c / \widetilde{c}$ does not depend on the choice of $\varphi$, since if we have two such $\varphi$ and $\psi, \nabla \varphi=k \nabla \psi$ for $k>0$.

Without loss of generality, we assume that $\widetilde{U} \subset[0, \pi]^{n}$. Then by Lemma 6.2 , one can reconstruct

$$
\int_{\mathbf{R}^{n-1}} e^{-i k \cdot x} V(x, y) d x, \quad \forall k \in \mathbf{Z}^{n-1} \backslash\{0\}
$$

from $\widetilde{\Lambda}_{V}$.

Now, the idea is to rotate and translate the domain $\widetilde{U}$ arbitrarily. Then $-\Delta+$ $V(X), X=\left(x, x_{n}\right)$, is transformed into $-\Delta+V\left(R X+X_{0}\right)$ with $R \in S O(n)$ and $X_{0} \in \mathbf{R}^{n}$, and we get the associated D-N map as the unitary transform of $\widetilde{\Lambda}_{V}$. We next imbed this IBVP into $\mathbf{H}^{n}$. By the above arguments, one can reconstruct $\hat{V}(R \Xi)$, where $\hat{V}(\Xi)$ is the Fourier transform of $V, R \in S O(n), \Xi=(k, \eta), \quad k \in$ $\mathbf{Z}^{n-1} \backslash\{0\}, \eta \in \mathbf{R}^{1}$. Since $S O(n)$ acts transitively on $S^{n-1}$, one can reconstruct $\hat{V}(\Xi)$ for $|\Xi|>1$ by varying $R$ and $\eta$. Since $\hat{V}(\Xi)$ is analytic, one can reconstruct $\hat{V}(\Xi)$ for all $\Xi \in \mathbf{R}^{n}$.

As is clear from the above arguments, the IBVP in $\mathbf{R}^{n}$ and that in $\mathbf{H}^{n}$ are equivalent. Therefore Theorem 6.3 also holds for a bounded contractible domain in $\mathbf{H}^{n}$ and also for any hyperbolic manifold.

6.4 Proof of Lemma 6.1. We prove Lemma 6.1 by showing the equivalence of IBVP and ISP. We follow the arguments of Isakov-Nachman [10]. 
Fix a constant $\lambda>0$ arbitrarily and let

$$
V(x, y)=\left\{\begin{array}{cc}
q(x, y)+\lambda, & (x, y) \in U, \\
0, & (x, y) \notin U .
\end{array}\right.
$$

Let $H(\theta)=H_{0}(\theta)+V$ be the Schrödinger operator on $\mathbf{E} \times \mathbf{R}$, and let $R(\lambda \pm i 0 ; \theta)=$ $(H(\theta)-\lambda \mp i 0)^{-1}$.

First let us note that

$$
R(\lambda \pm i 0 ; \theta) \in \mathbf{B}\left(H_{l o c}^{-s} ; H_{l o c}^{2-s}\right), \quad 0 \leq s \leq 2,
$$

which follows from the fact $R(\lambda \pm i 0 ; \theta) \in \mathbf{B}\left(L_{l o c}^{2} ; H_{l o c}^{2}\right) \cap \mathbf{B}\left(H_{l o c}^{-2} ; L_{l o c}^{2}\right)$ and interpolation. For $f \in L^{2}(S)$, we define $f_{S} \in H^{-1 / 2}$ by

$$
\left\langle\varphi, f_{S}\right\rangle=\int_{S} \varphi \bar{f} d S_{E}, \quad \varphi \in H^{1 / 2}
$$

where $d S_{E}$ is the surface element induced from the Euclidean metric $(d x)^{2}+(d y)^{2}$. Using (6.10) and (6.11), we have

$$
R(\lambda \pm i 0 ; \theta) f_{S} \in H_{l o c}^{3 / 2} .
$$

Let us introduce the boundary operator

$$
B(\theta) u=\left.\left(e^{2 y} \nu_{x} \cdot\left(\partial_{x}+i \theta\right) u+\nu_{y} \partial_{y} u\right)\right|_{S},
$$

where $\nu_{e}=\left(\nu_{x}, \nu_{y}\right)$ is the outer unit normal to $S$ with respect to the Euclidean metric $(d x)^{2}+(d y)^{2}$. For $u \in H_{\text {loc }}^{1 / 2}$, let $[u]_{\text {ext }}$ and $[u]_{\text {int }}$ be the boundary values of $u$ on $S$ from outside and inside of $S$, respectively.

Lemma 6.4. Let $f \in L^{2}(S)$. Then

$$
\left[B(\theta) R(\lambda+i 0 ; \theta) f_{S}\right]_{e x t}-\left[B(\theta) R(\lambda+i 0 ; \theta) f_{S}\right]_{\text {int }}=-\lambda\left(x, e^{y}\right) f,
$$

where $\lambda\left(x, x_{n}\right)$ is the function appearing in (6.9).

Proof. By the resolvent equation, we have

$$
R(\lambda+i 0 ; \theta) f_{S}=R_{0}(\lambda+i 0 ; \theta) f_{S}-R_{0}(\lambda+i 0 ; \theta) V R(\lambda+i 0 ; \theta) f_{S} .
$$

Therefore we have only to consider $u=R_{0}(\lambda+i 0 ; \theta) f_{S}$, since $R(\lambda+i 0 ; \theta) f_{S}-u \in$ $H_{l o c}^{2}$.

Take $\chi_{j} \in C_{0}^{\infty}(\mathbf{E} \times \mathbf{R})$ such that $\sum_{j} \chi_{j}=1$ near $S$, and each supp $\chi_{j}$ is contained in a local coordinate patch. Let $x_{n}=e^{y}, X=\left(x, x_{n}\right)$. Then we have

$$
-\Delta_{X}\left(\chi_{j} u\right)=x_{n}^{-2} \chi_{j} f_{s}+g_{j}, \quad g_{j} \in L^{2}
$$

Letting $F(X)$ be the fundamental solution to $-\Delta_{X}$, we have

$$
\chi_{j} u=\int F\left(X-X^{\prime}\right) x_{n}^{\prime}{ }^{-2} \chi_{j}\left(X^{\prime}\right) f\left(X^{\prime}\right) d S_{X^{\prime}}+h_{j}, \quad h_{j} \in H_{l o c}^{2} .
$$

Let $\widetilde{\nu}_{e}$ be the outer unit normal to $S$ with respect to the Euclidean metric $(d X)^{2}$. Then by the well-known computation from potential theory

$$
\left[\frac{\partial}{\partial \widetilde{\nu}_{e}}\left(\chi_{j} u\right)\right]_{e x t}-\left[\frac{\partial}{\partial \widetilde{\nu}_{e}}\left(\chi_{j} u\right)\right]_{i n t}=-x_{n}^{-2} \chi_{j} f
$$

Using (6.9), we get the lemma.

$$
\diamond
$$


Let $U_{\text {ext }}$ be the region exterior to $U$. Let $H_{\text {ext }}(\theta)=H_{0}(\theta)$ in $U_{\text {ext }}$ with Dirichlet boundary condition, and $R_{e x t}(z ; \theta)=\left(H_{e x t}(\theta)-z\right)^{-1}$. The limiting absorption principle also holds for $R_{\text {ext }}(z ; \theta)$ :

$$
R_{e x t}(\lambda \pm i 0 ; \theta) \in \mathbf{B}\left(L^{2, s} ; L^{2,-s}\right), \quad s>1 / 2 .
$$

For $f \in L^{2, s}, u_{\text {ext }}^{( \pm)}=R_{\text {ext }}(\lambda \pm i 0 ; \theta) f$ satisfies the radiation condition

$$
\left\{\begin{array}{c}
u_{e x t}^{( \pm)} \in L^{2,-s}, \\
F( \pm y>0)\left(i \partial_{y} \pm \sqrt{\lambda}\right) u_{e x t}^{(+)} \in L^{2,-\alpha} \\
F( \pm y>0)\left(i \partial_{y} \mp \sqrt{\lambda}\right) u_{e x t}^{(-)} \in L^{2,-\alpha}
\end{array}\right.
$$

for some $0<\alpha<1 / 2$. These facts will be proved in $\S 8$.

The D-N maps $\Lambda_{\text {ext }}^{( \pm)}(\theta)$ for the exterior Dirichlet problem are defined by

$$
\Lambda_{e x t}^{( \pm)}(\theta) f=\left.B(\theta) v_{e x t}^{( \pm)}\right|_{S},
$$

where $v_{\text {ext }}^{( \pm)}$are the outgoing or incoming solutions of

$$
\left(H_{0}(\theta)-\lambda\right) v=0 \quad \text { in } \quad U_{e x t},\left.\quad v\right|_{S}=f \in H^{3 / 2}(S),
$$

and where $B(\theta)$ is defined by (6.13) with the same $\nu_{e}$ as in (6.13). Therefore, viewed from $U_{e x t}, \nu_{e}$ is the inner unit normal.

Note that $v_{\text {ext }}^{( \pm)}$exist by virtue of the limiting absorption principle. In fact, take $\tilde{f} \in H^{2}\left(U_{\text {ext }}\right)$ such that $\tilde{f}=f$ on $S$ and $\tilde{f}$ has compact support. Then $v_{\text {ext }}^{( \pm)}$is given by

$$
v_{\text {ext }}^{( \pm)}=\tilde{f}+R_{\text {ext }}(\lambda \pm i 0 ; \theta)\left(\partial_{y}^{2}+e^{2 y}\left(\partial_{x}+i \theta\right)^{2}+\lambda\right) \tilde{f} .
$$

The uniqueness of outgoing or incoming solution will be proved in $\S 8$.

We need one more preparation. Let $u_{e x t} \in H_{l o c}^{2}\left(U_{\text {ext }}\right)$ and $u_{\text {int }} \in H^{2}(U)$ satisfy the equations

$$
\begin{gathered}
\left(H_{0}(\theta)-\lambda\right) u_{e x t}=0 \quad \text { in } \quad U_{\text {ext }},\left.\quad u_{e x t}\right|_{S}=f, \\
\left(H_{0}(\theta)+V-\lambda\right) u_{i n t}=0 \quad \text { in } \quad U,\left.\quad u_{i n t}\right|_{S}=f .
\end{gathered}
$$

Let $\chi_{\text {ext }}$ and $\chi_{\text {int }}$ be the characteristic functions of $U_{\text {ext }}$ and $U$, respectively. Letting $u=\chi_{e x t} u_{e x t}+\chi_{i n t} u_{i n t}$, we have for $\varphi \in C_{0}^{\infty}(\mathbf{E} \times \mathbf{R})$

$$
\int_{\mathbf{E} \times \mathbf{R}} u\left(H_{0}(\theta)+V-\lambda\right) \varphi d x d y=\int_{S}\left([B(\theta) u]_{i n t}-[B(\theta) u]_{e x t}\right) \varphi d S_{E} .
$$

Therefore we have

$$
u_{e x t}=R(\lambda \pm i 0 ; \theta)\left(\Lambda_{V-\lambda}(\theta)-\Lambda_{e x t}^{( \pm)}(\theta)\right) f
$$

accordingly as $u_{e x t}$ is outgoing or incoming.

For $f \in L^{2}(S)$, we define

$$
M_{S}^{( \pm)}(\theta) f=\left.\left(R(\lambda \pm i 0 ; \theta) f_{S}\right)\right|_{S} .
$$

Lemma 6.5. Suppose $\lambda \notin \sigma\left(H_{0}(\theta)_{D}+V\right)$. Then :

(1) $\Lambda_{V-\lambda}(\theta)-\Lambda_{e x t}^{( \pm)}(\theta): H^{3 / 2}(S) \rightarrow H^{1 / 2}(S)$ is an isomorphism.

(2) $\left(\Lambda_{V-\lambda}(\theta)-\Lambda_{e x t}^{( \pm)}(\theta)\right)^{-1}=M_{S}^{( \pm)}(\theta)$. 
Proof. We show that $M_{S}^{( \pm)}(\theta): H^{1 / 2}(S) \rightarrow H^{3 / 2}(S)$ is an isomorphism and

$$
M_{S}^{( \pm)}(\theta)\left(\Lambda_{V-\lambda}(\theta)-\Lambda_{e x t}^{( \pm)}(\theta)\right)=1 .
$$

Suppose $M_{S}^{( \pm)}(\theta) f=0$. Then $R(\lambda \pm i 0 ; \theta) f_{S}$ is a Dirichlet eigenfunction of $H_{0}(\theta)+V$ in the interior region, hence $R(\lambda \pm i 0 ; \theta) f=0$ on $U$. In the exterior region, $R(\lambda \pm i 0 ; \theta) f_{S}$ is a solution to the homogeneous Schrödinger equation satisfying the radiation condition. In $\S 8$, such a solution is shown to vanish identically. Therefore $R(\lambda \pm i 0 ; \theta) f_{S}=0$ on $U_{e x t}$. Hence by Lemma $6.5, f=0$, which proves that $M_{S}^{( \pm)}(\theta)$ is 1 to 1 . The formula (6.20) implies (6.22). This proves the lemma. $\diamond$.

Let us take notice of the following fact.

Lemma 6.6. For $f, g \in H^{3 / 2}(S)$

$$
\begin{aligned}
\left(\Lambda_{V-\lambda}(\theta) f, g\right)_{L^{2}(S)} & =\left(f, \Lambda_{V-\lambda}(\theta) g\right)_{L^{2}(S)}, \\
\left(\Lambda_{\text {ext }}^{( \pm)}(\theta) f, g\right)_{L^{2}(S)} & =\left(f, \Lambda_{\text {ext }}^{(\mp)}(\theta) g\right)_{L^{2}(S)} .
\end{aligned}
$$

Proof. We shall prove (6.24). Let $u$ be the outgoing solution of

$$
\left(H_{0}(\theta)-\lambda\right) u=0 \quad \text { in } U_{\text {ext }},\left.\quad u\right|_{S}=f,
$$

and $v$ the incoming solution of

$$
\left(H_{0}(\theta)-\lambda\right) v=0 \quad \text { in } \quad U_{e x t},\left.\quad v\right|_{S}=g .
$$

Let $U_{R}=\{(x, y) ;|y|<R\} \cap U_{\text {ext }}$. Then we have by integration by parts

$$
\begin{aligned}
& \int_{U_{R}}\left\{\left(H_{0}(\theta)-\lambda\right) u \bar{v}-u \overline{\left(H_{0}(\theta)-\lambda\right) v}\right\} d x d y \\
= & \left(\Lambda_{e x t}^{(+)}(\theta) f, g\right)_{L^{2}(S)}-\left(f, \Lambda_{e x t}^{(-)}(\theta) g\right)_{L^{2}(S)} \\
+ & \int_{y=R}\left\{u \overline{\left(\partial_{y} v+i \sqrt{\lambda} v\right)}-\left(\partial_{y} u-i \sqrt{\lambda} u\right) \bar{v}\right\} d x \\
- & \int_{y=-R}\left\{\left(\partial_{y} u+i \sqrt{\lambda} u\right) \bar{v}-u \overline{\left(\partial_{y} v-i \sqrt{\lambda} v\right)}\right\} d x .
\end{aligned}
$$

Since $u, v$ satisfy the radiation condition, we have

$$
\begin{gathered}
F( \pm y>0)\left(\partial_{y} u \mp i \sqrt{\lambda} u\right) \in L^{2, s-1}, \\
F( \pm y>0)\left(\partial_{y} v \pm i \sqrt{\lambda} v\right) \in L^{2, s-1}
\end{gathered}
$$

(see Theorem 7.7). Therefore the integrands in $\int_{y=R} \cdots d x$ and $\int_{y=-R} \cdots d x$ belong to

$L^{1}\left(U_{\text {ext }} ;\langle y\rangle^{-1} d x d y\right)$. We then see that these integrals vanish as $R \rightarrow \infty$ along a suitable sequence $\left\{R_{j}\right\}$. This proves (6.24). The formula (6.23) is proved by the similar and simpler method of integration by parts.

Let us introduce the transformation from near field pattern to far field pattern. Let $\mathcal{F}^{( \pm)}(\lambda ; \theta)$ be defined by (4.14). Then one can naturally define

$$
\mathcal{G}^{( \pm)}(\lambda ; \theta)=\mathcal{F}^{( \pm)}(\lambda ; \theta)\left(\Lambda_{V-\lambda}(\theta)-\Lambda_{\text {ext }}^{( \pm)}(\theta)\right)
$$

Using Lemma 6.6 (2), we have

\section{Lemma 6.7.}

$$
\mathcal{G}^{( \pm)}(\lambda ; \theta) M_{S}^{( \pm)}(\theta)=\mathcal{F}^{( \pm)}(\lambda ; \theta)
$$


By virtue of (6.20), Theorem 4.4 and Lemma 6.7, we have

Lemma 6.8. Let $u_{\text {ext }}^{( \pm)}$be the outgoing or incoming solution to (6.17). Then as $y \rightarrow-\infty$

$$
u_{e x t}^{( \pm)} \sim C_{ \pm}(\lambda) e^{\mp i \sqrt{\lambda} y} \mathcal{G}^{( \pm)}(\lambda ; \theta) f \quad \text { in } \quad L^{2}(\mathbf{E})
$$

where $C_{ \pm}(\lambda)$ is given in Theorem 4.4 .

Finally we introduce the generalized Fourier transformation associated with $H_{0}(\theta)$ in the exterior domain. It follows easily from Lemma $6.6(2)$ that

$$
R_{e x t}(\lambda \pm i 0 ; \theta)=R_{0}(\lambda \pm i 0 ; \theta)-R(\lambda \pm i 0 ; \theta)\left(\Lambda_{V-\lambda}(\theta)-\Lambda_{\text {ext }}^{( \pm)}(\theta)\right) R_{0}(\lambda \pm i 0 ; \theta)
$$

Taking the adjoint and using Lemma 6.7, we also have

$$
R_{\text {ext }}(\lambda \pm i 0 ; \theta)=R_{0}(\lambda \pm i 0 ; \theta)-R_{0}(\lambda \pm i 0 ; \theta)\left(\Lambda_{V-\lambda}(\theta)-\Lambda_{\text {ext }}^{( \pm)}(\theta)\right) R(\lambda \pm i 0 ; \theta) \text {. }
$$

We put

$$
\mathcal{F}_{\text {ext }}^{(-)}(\lambda ; \theta)=\mathcal{F}_{0}^{(-)}(\lambda ; \theta)-\mathcal{F}_{0}^{(-)}(\lambda ; \theta)\left(\Lambda_{V-\lambda}(\theta)-\Lambda_{\text {ext }}^{(-)}(\theta)\right) R(\lambda-i 0 ; \theta) .
$$

Then by (6.27) we have as $y \rightarrow-\infty$

$$
R_{\text {ext }}(\lambda-i 0 ; \theta) f \sim C_{-}(\lambda) e^{i \sqrt{\lambda} y} \mathcal{F}_{\text {ext }}^{(-)}(\lambda ; \theta) f .
$$

This shows in particular that $\mathcal{F}_{\text {ext }}^{(-)}(\lambda ; \theta)$ does not depend on $V$. It is not hard to show that for $\varphi \in L^{2}(\mathbf{E})$

$$
\begin{gathered}
\left(H_{0}(\theta)-\lambda\right) \mathcal{F}_{\text {ext }}^{(-)}(\lambda ; \theta)^{*} \varphi=0 \text { in } U_{\text {ext }}, \\
\mathcal{F}_{\text {ext }}^{(-)}(\lambda ; \theta)^{*} \varphi=0 \text { on } S, \\
\mathcal{F}_{\text {ext }}^{(-)}(\lambda ; \theta)^{*} \varphi-\mathcal{F}_{0}^{(-)}(\lambda ; \theta)^{*} \varphi \text { is outgoing. }
\end{gathered}
$$

We define the geometric scattering amplitude for the exterior domain by

$$
\widetilde{A_{e x t}}(\lambda ; \theta)=\mathcal{F}^{(+)}(\lambda ; \theta)\left(\Lambda_{V-\lambda}(\theta)-\Lambda_{\text {ext }}^{(+)}(\theta)\right) \mathcal{F}_{0}^{(-)}(\lambda ; \theta)^{*}
$$

Then we have as $y \rightarrow-\infty$

$$
\mathcal{F}_{\text {ext }}^{(-)}(\lambda ; \theta)^{*} \varphi-\mathcal{F}_{0}^{(-)}(\lambda ; \theta)^{*} \varphi \sim-C_{+}(\lambda) e^{-i \sqrt{\lambda} y} \widetilde{A_{\text {ext }}}(\lambda ; \theta) \varphi .
$$

\section{Lemma 6.9.}

$$
\mathcal{G}^{(+)}(\lambda ; \theta) M_{S}^{(+)}(\theta) \mathcal{G}^{(-)}(\lambda ; \theta)^{*}=\widetilde{A_{e x t}}(\lambda ; \theta)-\widetilde{A}(\lambda ; \theta)
$$

Proof. Let $u=\mathcal{F}^{(-)}(\lambda ; \theta)^{*} \varphi-\mathcal{F}_{\text {ext }}^{(-)}(\lambda ; \theta)^{*} \varphi$. Then since $u$ is the outgoing solution of

$$
\left(H_{0}(\theta)-\lambda\right) u=0 \quad \text { in } \quad U_{e x t},\left.\quad u\right|_{S}=\mathcal{F}^{(-)}(\lambda ; \theta)^{*} \varphi
$$

we have by $(6.20)$

$$
\begin{aligned}
u & =R(\lambda+i 0 ; \theta)\left(\Lambda_{V-\lambda}(\theta)-\Lambda_{\text {ext }}^{(+)}(\theta)\right) \mathcal{F}^{(-)}(\lambda ; \theta)^{*} \varphi \\
& =R(\lambda+i 0 ; \theta) \mathcal{G}^{(-)}(\lambda ; \theta)^{*} \varphi
\end{aligned}
$$

Therefore as $y \rightarrow-\infty$

$$
u \sim C_{+}(\lambda) e^{-i \sqrt{\lambda} y} \mathcal{F}^{(+)}(\lambda ; \theta) \mathcal{G}^{(-)}(\lambda ; \theta)^{*} \varphi .
$$

Inserting $1=\left(\Lambda_{V-\lambda}(\theta)-\Lambda_{e x t}^{(+)}(\theta)\right) M_{S}^{(+)}(\theta)$, we obtain

$$
u \sim C_{+}(\lambda) e^{-i \sqrt{\lambda} y} \mathcal{G}^{(+)}(\lambda ; \theta) M_{S}^{(+)}(\theta) \mathcal{G}^{(-)}(\lambda ; \theta)^{*} \varphi .
$$


On the other hand, we have

$$
\mathcal{F}^{(-)}(\lambda ; \theta)^{*} \varphi-\mathcal{F}_{\text {ext }}^{(-)}(\lambda ; \theta)^{*} \varphi \sim-C_{+}(\lambda) e^{-i \sqrt{\lambda} y}\left(\widetilde{A}(\lambda ; \theta) \varphi-\widetilde{A_{\text {ext }}}(\lambda ; \theta) \varphi\right) .
$$

Comparing (6.32) and (6.33), we obtain the lemma.

We prove the equivalence of IBVP and ISP by showing that $\Lambda_{V-\lambda}(\theta)$ and $A(\lambda ; \theta)$ determine each other.

Given $\Lambda_{V-\lambda}(\theta)$, construct $M_{S}^{(+)}(\theta)$ by Lemma 6.5 . Then $A(\lambda ; \theta)$ is constructed by Lemma 6.9 .

The converse direction is less explict. We show that $\mathcal{G}^{( \pm)}(\lambda ; \theta)$ are 1 to 1 . Then, since the ranges of $\mathcal{G}^{( \pm)}(\lambda ; \theta)^{*}$ are dense in $L^{2}(\mathbf{E})$, Lemma 6.10 gives $\Lambda_{V-\lambda}(\theta)$ from $A(\lambda ; \theta)$.

Suppose $\mathcal{G}^{( \pm)}(\lambda ; \theta) f=0$. Let $u$ be the (outgoing or incoming) solution to (6.17). By virtue of Lema 6.9 , we have $\|u(\cdot, y)\|_{L^{2}(\mathbf{E})} \rightarrow 0$ as $y \rightarrow-\infty$. By expanding $u$ into a Fourier series $u=\frac{1}{|\mathbf{E}|} \sum \hat{u}\left(\gamma^{*}, y\right) e^{i \gamma^{*} \cdot x}$, we see that the Fourier coefficient $\hat{u}\left(\gamma^{*}, y\right)$ satisfies the equation $-v^{\prime \prime}+e^{2 y}\left(\gamma^{*}+\theta\right)^{2} v-\lambda v=0$. Therefore $\hat{u}\left(\gamma^{*}, y\right)$ is written by a linear combination of modified Bessel functions. Since $\hat{u}\left(\gamma^{*}, y\right) \rightarrow 0$ as $y \rightarrow-\infty$, we have $\hat{u}\left(\gamma^{*}, y\right)=0$ by observing the behavior of $K_{i \sqrt{\lambda}}\left(\left|\gamma^{*}+\theta\right| e^{y}\right), I_{i \sqrt{\lambda}}\left(\left|\gamma^{*}+\theta\right| e^{y}\right)$ as $y \rightarrow-\infty$. Then $u(x, y)=0$ for $y<-R, R$ being sufficiently large. By the unique continuation theorem, $u$ vanishes identically on $U_{\text {ext }}$. Therefore $f=0$.

6.5 ISP at the cusp. We show that the D-N map and the scattering amplitude at the cusp determine each other. As in (6.25) we introduce a transformation from near field pattern to far field pattern at the cusp. We put

$$
\Lambda_{V-\lambda}=\Lambda_{V-\lambda}(0), \quad \Lambda_{e x t}^{( \pm)}=\Lambda_{\text {ext }}^{( \pm)}(0), \quad R(\lambda \pm i 0)=R(\lambda \pm i 0 ; 0)
$$

and define

$$
\mathcal{G}_{c \gamma^{*}}^{( \pm)}(\lambda)=\mathcal{F}_{c \gamma^{*}}^{( \pm)}(\lambda)\left(\Lambda_{V-\lambda}-\Lambda_{e x t}^{( \pm)}\right)
$$

More precisely, for $f \in H^{3 / 2}(S)$ we put

$$
\begin{gathered}
u_{ \pm}=(1-V R(\lambda \pm i 0))\left(\Lambda_{V-\lambda}-\Lambda_{e x t}^{( \pm)}\right) f \\
\mathcal{G}_{c \gamma^{*}}^{( \pm)}(\lambda) f=\int_{S} I_{\mp i \sqrt{\lambda}}\left(\left|\gamma^{*}\right| e^{y}\right) e^{-i \gamma^{*} \cdot x} u_{ \pm}(x, y) d S \quad\left(\gamma^{*} \neq 0\right), \\
\mathcal{G}_{c \gamma^{*}}^{( \pm)}(\lambda) f=(2 \pi)^{-1 / 2} \int_{S} e^{\mp i \sqrt{\lambda} y} u_{ \pm}(x, y) d S \quad\left(\gamma^{*}=0\right) .
\end{gathered}
$$

By Lemma 6.5 (2), we have

Lemma 6.10. $\mathcal{G}_{c \gamma^{*}}^{( \pm)}(\lambda) M_{S}^{( \pm)}=\mathcal{F}_{c \gamma^{*}}^{( \pm)}(\lambda)$, where $M_{S}^{( \pm)}=M_{S}^{( \pm)}(0)$.

By virtue of Theorem 4.11, we also have

Lemma 6.11. Let $u_{\text {ext }}^{( \pm)}$be the outgoing or incoming solution to (6.17). Then as $y \rightarrow \infty$

$$
\begin{gathered}
\left\langle e^{-i \gamma^{*} \cdot x}, u_{e x t}^{( \pm)}\right\rangle \sim \sqrt{\frac{\pi}{2\left|\gamma^{*}\right|}} \exp \left(-\frac{y}{2}-\left|\gamma^{*}\right| e^{y}\right) \mathcal{G}_{c \gamma^{*}}^{( \pm)}(\lambda) f \quad\left(\gamma^{*} \neq 0\right), \\
\left\langle e^{-i \gamma^{*} \cdot x}, u_{e x t}^{( \pm)}\right\rangle \sim \mp i \sqrt{\frac{\pi}{2 \lambda}} e^{ \pm i \sqrt{\lambda} y} \mathcal{G}_{c \gamma^{*}}^{( \pm)}(\lambda) f \quad\left(\gamma^{*}=0\right) .
\end{gathered}
$$


We also introduce the generlized Fourier transformation associated with the cusp. We put

$$
\begin{aligned}
& \mathcal{F}_{\text {ext }, c \gamma^{*}}^{(-)}(\lambda)=\mathcal{F}_{0 c \gamma^{*}}^{(-)}(\lambda)\left[1-\left(\Lambda_{V-\lambda}-\Lambda_{\text {ext }}^{(-)}\right) R(\lambda-i 0)\right], \\
& \mathcal{F}_{\text {ext }, c}^{(-)}(\lambda)=\sum_{\gamma^{*} \in \Gamma^{*}} \mu_{\gamma^{*}} e^{i \gamma^{*} \cdot x} \mathcal{F}_{\text {ext }, c \gamma^{*}}^{(-)}(\lambda), \\
& \mathcal{G}_{c}^{( \pm)}(\lambda)=\sum_{\gamma^{*} \in \Gamma^{*}} \mu_{\gamma^{*}} e^{i \gamma^{*} \cdot x} \mathcal{G}_{c \gamma^{*}}^{( \pm)}(\lambda) .
\end{aligned}
$$

We finally put

$$
\widetilde{A_{e x t, c}}(\lambda)=\mathcal{F}_{c}^{(+)}(\lambda)\left(\Lambda_{V-\lambda}-\Lambda_{e x t}^{(+)}\right) \mathcal{F}_{0 c}^{(-)^{*}}(\lambda)
$$

Then as in Lemma 6.9, we have

Lemma 6.12. $\mathcal{G}_{c}^{(+)}(\lambda) M_{S}^{(+)} \mathcal{G}_{c}^{(-)}(\lambda)^{*}=\widetilde{A_{\text {ext }, c}}(\lambda)-\widetilde{A}_{c}(\lambda)$.

Proof. One can show that for $\varphi \in L^{2}(\mathbf{E})$

$$
\begin{gathered}
\left(H_{0}-\lambda\right) \mathcal{F}_{\text {ext,c }}^{(-)}(\lambda)^{*} \varphi=0 \quad \text { in } \quad U_{\text {ext }}, \\
\mathcal{F}_{\text {ext }, c}^{(-)}(\lambda)^{*} \varphi=0 \text { on } S, \\
\mathcal{F}_{\text {ext }, c}^{(-)}(\lambda)^{*} \varphi-\mathcal{F}_{0 c}^{(-)}(\lambda)^{*} \varphi \quad \text { is outgoing. }
\end{gathered}
$$

Let $u=\mathcal{F}_{c}^{(-)}(\lambda)^{*} \varphi-\mathcal{F}_{\text {ext,c }}^{(-)}(\lambda)^{*} \varphi$. Then as in the proof of Lemma 6.9,

$$
\begin{aligned}
u & =R(\lambda+i 0)\left(\Lambda_{V-\lambda}-\Lambda_{e x t}^{(+)}\right) \mathcal{F}_{c}^{(-)}(\lambda)^{*} \varphi \\
& =R(\lambda+i 0) \mathcal{G}_{c}^{(-)}(\lambda)^{*} \varphi .
\end{aligned}
$$

If $0 \neq \gamma^{*} \in \Gamma^{*}$, we have as $y \rightarrow \infty$

$$
\begin{aligned}
\left\langle e^{-i \gamma^{*} \cdot x}, u\right\rangle & \sim \sqrt{\frac{\pi}{2\left|\gamma^{*}\right|}} \exp \left(-\frac{y}{2}-\left|\gamma^{*}\right| e^{y}\right) \mathcal{F}_{c \gamma^{*}}^{(+)}(\lambda) \mathcal{G}_{c}^{(-)}(\lambda)^{*} \varphi \\
& \sim \sqrt{\frac{\pi}{2\left|\gamma^{*}\right|}} \exp \left(-\frac{y}{2}-\left|\gamma^{*}\right| e^{y}\right) \mathcal{G}_{c \gamma^{*}}^{(+)}(\lambda) M_{S}^{(+)} \mathcal{G}_{c}^{(-)}(\lambda)^{*} \varphi
\end{aligned}
$$

On the other hand, we have

$$
\begin{gathered}
\left\langle e^{-i \gamma^{*} \cdot x}, \mathcal{F}_{c}^{(-)}(\lambda)^{*} \varphi-\mathcal{F}_{\text {ext }, c}^{(-)}(\lambda)^{*} \varphi\right\rangle \\
\sim-\sqrt{\frac{\pi}{2\left|\gamma^{*}\right|}} \exp \left(-\frac{y}{2}-\left|\gamma^{*}\right| e^{y}\right)\left(\widetilde{A_{c}}(\lambda) \varphi-\widetilde{A_{\text {ext }, c}}(\lambda) \varphi\right) .
\end{gathered}
$$

By computing the case $\gamma^{*}=0$ also, and comparing the asmptotic expansions, we get the lemma.

We now show that $\Lambda_{V-\lambda}$ and $\widetilde{A_{c}}(\lambda)$ determine each other. By Lemma 6.12, $\widetilde{A_{c}}(\lambda)$ is constructed from $\Lambda_{V-\lambda}$. To show the converse direction, we show that $\mathcal{G}_{c}^{( \pm)}(\lambda)$ are 1 to 1 . Suppose $\mathcal{G}_{c}^{( \pm)}(\lambda) f=0$. Let $u$ be the (outgoing or incoming) solution to $\left(H_{0}-\lambda\right) u=0$ in $U_{\text {ext }}, u=f$ on $S$. We expand $u$ into a Fourier series $u=\frac{1}{|\mathbf{E}|} \sum \hat{u}\left(\gamma^{*}, y\right) e^{i \gamma^{*} \cdot x}$. Then $\hat{u}\left(\gamma^{*}, y\right)$ satisfies $-v^{\prime \prime}+e^{2 y}\left|\gamma^{*}\right|^{2}-\lambda v=0$ for large $|y|$, hence is written as a linear combination of modified Bessel functions (or of $e^{ \pm i \sqrt{\lambda} y}$ if $\left.\gamma^{*}=0\right)$. In view of Lemma 6.12 and $(2.5),(2.6), \hat{u}\left(\gamma^{*}, y\right)=0$ for large $y$. By the unique continuation theorem $u=0$ on $U_{\text {ext }}$, hence $f=0$. 


\section{Resolvent estimtes for the 1-Dimensional PROBlem}

We shall prove the limiting absorption principle for $L_{0}(\zeta)$ defined by (2.11). We prepare a-priori estimates (Lemmas $7.1 \sim 7.4$ ) and the uniqueness result (Lemma 7.6). We fix a compact interval $I \subset(0, \infty)$ and let $J=\{z \in \mathbf{C} ; \operatorname{Re} z \in I,|\operatorname{Im} z| \leq$ 1 . In the following, $C$ denotes a constant independent of $\zeta \geq 0$ and $z \in J$. In this section we take the branch of $\sqrt{z}$ such that $\operatorname{Im} \sqrt{z} \geq 0$.

Lemma 7.1. Let u satisfy

$$
-u^{\prime \prime}+\left(e^{2 y} \zeta^{2}-z\right) u=f, \quad \zeta \geq 0
$$

If $u \in L^{2, s}, f \in L^{2, s}$, for some $s \in \mathbf{R}$, we have

$$
\left\|u^{\prime}\right\|_{s}+\left\|e^{y} \zeta u\right\|_{s} \leq C\left(\|u\|_{s}+\|f\|_{s}\right) .
$$

Proof. The proof is based on the following formula

$$
\begin{gathered}
\int_{a}^{b} \varphi\left(-u^{\prime \prime}+\left(V_{0}-z\right) u\right) \bar{u} d y \\
=-\left[\varphi u^{\prime} \bar{u}\right]_{a}^{b}+\int_{a}^{b} \varphi^{\prime} u^{\prime} \bar{u} d y+\int_{a}^{b} \varphi\left|u^{\prime}\right|^{2} d y+\int_{a}^{b} \varphi\left(V_{0}-z\right)|u|^{2} d y,
\end{gathered}
$$

where $\varphi$ is real-valued.

Let $V_{0}=e^{2 y} \zeta^{2}$ and take the real part of (7.1). Then we have

$$
\begin{gathered}
\int_{-R}^{R} \varphi\left|u^{\prime}\right|^{2} d y+\int_{-R}^{R} \varphi e^{2 y} \zeta^{2}|u|^{2} d y \\
=\operatorname{Re} \int_{-R}^{R} \varphi f \bar{u} d y+\frac{1}{2} \int_{-R}^{R} \varphi^{\prime \prime}|u|^{2} d y+\operatorname{Re} z \int_{-R}^{R} \varphi|u|^{2} d y \\
+\operatorname{Re}\left[\varphi u^{\prime} \bar{u}\right]_{-R}^{R}-\frac{1}{2}\left[\varphi^{\prime}|u|^{2}\right]_{-R}^{R} .
\end{gathered}
$$

Taking $\varphi=\langle y\rangle^{2 s}$, we have

$$
\begin{gathered}
\int_{-R}^{R} \varphi\left|u^{\prime}\right|^{2} d y+\int_{-R}^{R} \varphi e^{2 y} \zeta^{2}|u|^{2} d y \\
\leq C\left(\|f\|_{s}^{2}+\|u\|_{s}^{2}\right)+\operatorname{Re}\left[\varphi u^{\prime} \bar{u}\right]_{-R}^{R}-\frac{1}{2}\left[\varphi^{\prime}|u|^{2}\right]_{-R}^{R} .
\end{gathered}
$$

The lemma is then proved if we show

$$
\begin{gathered}
u \in L^{2, s} \Longrightarrow \liminf _{R \rightarrow \infty} R^{2 s+1}\left(|u(R)|^{2}+|u(-R)|^{2}\right)=0, \\
u \in L^{2, s} \Longrightarrow \liminf _{R \rightarrow \infty}\left[\varphi u^{\prime} \bar{u}\right]_{-R}^{R} \leq 0 .
\end{gathered}
$$

The fisrt assertion is obvious. To show (7.3), first note

$$
\operatorname{Re}\left[\varphi u^{\prime} \bar{u}\right]_{-R}^{R}=\frac{1}{2}\left[\left(\varphi|u|^{2}\right)^{\prime}\right]_{-R}^{R}-\frac{1}{2}\left[\varphi^{\prime}|u|^{2}\right]_{-R}^{R} .
$$

As above $\liminf _{R \rightarrow \infty}\left|\left[\varphi^{\prime}|u|^{2}\right]_{-R}^{R}\right|=0$. If $\liminf _{R \rightarrow \infty}\left[\left(\varphi|u|^{2}\right)^{\prime}\right]_{-R}^{R} \geq 2 \delta>0$, there exists $R_{0}>0$ such that

$$
\left[\left(\varphi|u|^{2}\right)^{\prime}\right]_{-R}^{R} \geq \delta \quad \text { for } \quad R \geq R_{0} .
$$

Integrate over $\left(R_{0}, R\right)$ to get

$$
\varphi(R)|u(R)|^{2}+\varphi(-R)|u(-R)|^{2}-\varphi\left(R_{0}\right)\left|u\left(R_{0}\right)\right|^{2}-\varphi\left(-R_{0}\right)\left|u\left(-R_{0}\right)\right|^{2} \geq \delta\left(R-R_{0}\right) .
$$


Take $\liminf \operatorname{in}_{R \rightarrow \infty}$ to arrive at a contradiction.

Lemma 7.2. Let $u$ satisfy

$$
-u^{\prime \prime}+\left(e^{2 y} \zeta^{2}-z\right) u=f, \quad \zeta \geq 0
$$

If $u \in L^{2, s}, f \in L^{2, s+1}$, for some $s \in \mathbf{R}$, and $\operatorname{Im} \sqrt{z}>0$, we have

$$
\operatorname{Im} \sqrt{z}\|u\|_{s+1 / 2} \leq C\left(\|u\|_{s}+\|f\|_{s+1}\right) .
$$

Proof. Take $\varphi=\langle y\rangle^{2 s+1}$ and compute

$$
\operatorname{Im} \int_{-R}^{R} \varphi f \bar{u} d y=-\operatorname{Im}\left[\varphi u^{\prime} \bar{u}\right]_{-R}^{R}+\operatorname{Im} \int_{-R}^{R} \varphi^{\prime} u^{\prime} \bar{u} d y-\operatorname{Im} z \int_{-R}^{R} \varphi|u|^{2} d y .
$$

The lemma then follows from Lemma 7.1.

Let

$$
D_{ \pm}=\sqrt{z} \pm i \partial_{y}
$$

Lemma 7.3. Let $u$ satisfy $-u^{\prime \prime}+\left(e^{2 y} \zeta^{2}-z\right) u=f$.

(1) If $\zeta \geq 0, u \in L^{2,-s}, f \in L^{2, s}$ for some $s>1 / 2$, Im $\sqrt{z} \geq 0$, and $F(y<$ -1) $D_{-} u \in L^{2, s-1}$, we have

$$
\left\|F(y<-1) D_{-} u\right\|_{s-1} \leq C\left(\|u\|_{-s}+\|f\|_{s}\right) .
$$

(2) If $\zeta=0, u \in L^{2,-s}, f \in L^{2, s}$ for some $s>1 / 2, \operatorname{Im} \sqrt{z} \geq 0$, and $F(y>1) D_{+} u \in$ $L^{2, s-1}$, we have

$$
\left\|F(y>1) D_{+} u\right\|_{s-1} \leq C\left(\|u\|_{-s}+\|f\|_{s}\right) .
$$

Proof. The differential equation is rewritten as

$$
\left(D_{-} u\right)^{\prime}=i \sqrt{z} D_{-} u-i e^{2 y} \zeta^{2} u+i f .
$$

Let $\varphi(y)=0(y>0), \varphi(y)=(-y)^{2 s-1}(y<-1)$. Multiply $(7.5)$ by $\varphi(y) \overline{D_{-} u}$, integrate over $(-R, 0)$ and take the real part. Then we have

$$
\begin{aligned}
-\frac{1}{2} \int_{-R}^{0} \varphi^{\prime}\left|D_{-} u\right|^{2} d y= & -\frac{1}{2}\left[\varphi\left|D_{-} u\right|^{2}\right]_{-R}^{0}-\operatorname{Im} \sqrt{z} \int_{-R}^{0} \varphi\left|D_{-} u\right|^{2} d y \\
& -\operatorname{Re} i \int_{-R}^{0} \varphi e^{2 y} \zeta^{2} u \overline{D_{-} u} d y+\operatorname{Re} i \int_{-R}^{0} \varphi f \overline{D_{-} u} d y .
\end{aligned}
$$

Let $R \rightarrow \infty$ (along a suitable sequence). Applying the Schwarz inequality to the last term of the right-hand side, we have

$$
C_{1}\left\|F(y<-1) D_{-} u\right\|_{s-1}^{2} \leq C_{2}\left(\|f\|_{s}^{2}+\|u\|_{-s}^{2}\right)-\operatorname{Re} i \int_{-\infty}^{0} \varphi e^{2 y} \zeta^{2} u \overline{D_{-} u} d y
$$

for some constants $C_{1}, C_{2}>0$. Again by integration by parts we have

$$
-\operatorname{Re} i \int_{-\infty}^{0} \varphi e^{2 y} \lambda u \overline{D_{-} u} d y=-\operatorname{Im} \sqrt{z} \int_{-\infty}^{0} \varphi e^{2 y} \zeta^{2}|u|^{2} d y-\frac{\zeta^{2}}{2} \int_{-\infty}^{0}\left(\varphi e^{2 y}\right)^{\prime}|u|^{2} d y .
$$

There exists $N>0$ such that $\left(\varphi e^{2 y}\right)^{\prime}>0$ for $y<-N$. Therefore this integral is dominated from above by

$$
C_{3} \int_{-\infty}^{0} \zeta^{2} e^{2 y}|u|^{2} d y \leq C_{4}\left(\|u\|_{-s}^{2}+\|f\|_{s}^{2}\right),
$$

where we have used Lemma 7.1. This proves (1). The assertion (2) is proved similarly. 
Lemma 7.4. Let $u$ satisfy $-u^{\prime \prime}+\left(e^{2 y} \zeta^{2}-z\right) u=f$. Suppose $\operatorname{Im} \sqrt{z}>0$, and $u \in L^{2,-s}, f \in L^{2, s}$ for some $s>1 / 2$. Let $s=1 / 2+\epsilon$. Then

$$
\|F(y<-r) u\|_{-s}+\|F(y>r) u\|_{-s} \leq C r^{-\epsilon / 2}\left(\|u\|_{-s}+\|f\|_{s}\right) .
$$

Proof. We first show for $y<0$,

$$
-\operatorname{Im} u^{\prime}(y) \overline{u(y)} \leq C\langle y\rangle^{\epsilon}\left(\|u\|_{-s}^{2}+\|f\|_{s}^{2}\right) .
$$

In fact by (7.1)

$$
-\left[u^{\prime}(\varphi \bar{u})\right]_{-R}^{y}=\int_{-R}^{y} \varphi f \bar{u} d t-\int_{-R}^{y}\left(e^{2 t} \zeta^{2}-z\right) \varphi|u|^{2} d t-\int_{-R}^{y} \varphi^{\prime} u^{\prime} \bar{u} d t-\int_{-R}^{y} \varphi\left|u^{\prime}\right|^{2} d t .
$$

Taking the imaginary part, we have

$$
-\operatorname{Im}\left[u^{\prime} \varphi \bar{u}\right]_{-R}^{y}=\operatorname{Im} \int_{-R}^{y} \varphi f \bar{u} d t+\operatorname{Im} z \int_{-R}^{y} \varphi|u|^{2} d t-\operatorname{Im} \int_{-R}^{y} \varphi^{\prime} u^{\prime} \bar{u} d t .
$$

Put $\varphi=\langle y\rangle^{-1-2 \epsilon}$ and let $R \rightarrow \infty$. Then by using Lemma 7.2, we have

$$
\begin{aligned}
& -\operatorname{Im} \varphi(y) u^{\prime}(y) \overline{u(y)} \\
\leq & \langle y\rangle^{-1-2 \epsilon}\|f\|_{s}\|u\|_{-s}+\operatorname{Im} z\langle y\rangle^{-1-\epsilon}\|u\|_{-\epsilon}^{2}+C\langle y\rangle^{-1-\epsilon}\left\|u^{\prime}\right\|_{-1-\epsilon}\|u\|_{-1-\epsilon} \\
\leq & C\langle y\rangle^{-1-\epsilon}\left(\|u\|_{-s}^{2}+\|f\|_{s}^{2}\right),
\end{aligned}
$$

which proves (7.6).

Since

we have by (7.6)

$$
(\operatorname{Re} \sqrt{z})|u|^{2}=\operatorname{Re}\left(D_{-} u\right) \bar{u}-\operatorname{Im} u^{\prime} \bar{u}
$$

$$
(\operatorname{Re} \sqrt{z})|u|^{2} \leq\left|D_{-} u\right|^{2}+C\langle y\rangle^{\epsilon}\left(\|u\|_{-s}^{2}+\|f\|_{s}^{2}\right) .
$$

Multiply $\langle y\rangle^{-1-2 \epsilon}$ and integrate over $(-\infty,-r)$. Then

$$
\operatorname{Re} \sqrt{z} \int_{-\infty}^{-r}\langle y\rangle^{-1-2 \epsilon}|u|^{2} d y \leq C r^{-\epsilon} \int_{-\infty}^{-r}\langle y\rangle^{-1-\epsilon}\left|D_{-} u\right|^{2} d y+C r^{-\epsilon}\left(\|u\|_{-s}^{2}+\|f\|_{s}^{2}\right) .
$$

This proves the lemma for $F(y<-r) u$. The estimate for $F(y>r) u$ is proved similarly.

Definition 7.5. A solution $u$ of the equation $-u^{\prime \prime}+e^{2 y} \zeta^{2} u-E u=f(E>0)$ is said to satisfy the outgoing radiation condition if for some $0<\alpha<1 / 2<s$

$$
u \in L^{2,-s}, \quad F( \pm y>0)\left(i \partial_{y} \pm \sqrt{E}\right) u \in L^{2,-\alpha} .
$$

Lemma 7.6. An outgoing solution of $-u^{\prime \prime}+e^{2 y} \zeta^{2} u-E u=0, E>0$, vanishes identically.

Proof. For $R>2$, pick $\chi_{R}(y) \in C_{0}^{\infty}(\mathbf{R})$ such that $\chi_{R}(-y)=-\chi_{R}(y), \chi_{R}(y)=0$ if $0<y<1$ or $y>R+1, \chi_{R}(y)=1$ if $2<y<R, \chi_{R}(y) \geq 0$ for $y \geq 0$. Put

$$
\tilde{\chi}_{R}(y)=\int_{-\infty}^{y} \chi_{R}(t)\langle t\rangle^{-2 \alpha} d t \in C_{0}^{\infty}(\mathbf{R}) \text {. }
$$

Taking the imaginary part of $0=\left(-u^{\prime \prime}+e^{2 y} \zeta^{2} u-E u, \widetilde{\chi}_{R} u\right)$, we get

$$
\operatorname{Im}\left(u^{\prime}, \chi_{R}\langle y\rangle^{-2 \alpha} u\right)=0 .
$$

Letting $f=u^{\prime}-i \sqrt{E}(\operatorname{sgn} y) u$, we have

$$
\sqrt{E}\left(\left|\chi_{R}\right|\langle y\rangle^{-2 \alpha} u, u\right)=-\operatorname{Im}\left(f, \chi_{R}\langle y\rangle^{-2 \alpha} u\right) .
$$


We have, therefore,

$$
\sqrt{E}\left\|\sqrt{\left|\chi_{R}\right|} u\right\|_{-\alpha} \leq\left\|\sqrt{\left|\chi_{R}\right|} f\right\|_{-\alpha} .
$$

Letting $R \rightarrow \infty$, we get $u \in L^{2,-\alpha}$. Now we use Theorem 9.1 (letting $B(t)=e^{-2 t} \zeta^{2}$ and $V(t)=0)$ to show $u=0 . \quad \diamond$

We also say that a solution of the equation $-u^{\prime \prime}+e^{2 y} \zeta^{2} u-E u=f(E>0)$ satisfies the incoming radiation condition if

$$
u \in L^{2,-s}, \quad F( \pm y>0)\left(i \partial_{y} \mp \sqrt{E}\right) u \in L^{2,-\alpha}
$$

for some $0<\alpha<1 / 2<s$. Lemma 7.6 also holds for the incoming solution.

Theorem 7.7. For $E>0, \zeta \geq 0$ and $s>1 / 2$, there exists the strong limit

$$
\mathrm{s}-\lim _{\epsilon \downarrow 0}\left(-\partial_{y}{ }^{2}+e^{2 y} \zeta^{2}-E \mp i \epsilon\right)^{-1} \in \mathbf{B}\left(L^{2, s} ; L^{2,-s}\right) .
$$

Moreover for $f \in L^{2, s},\left(-\partial_{y}{ }^{2}+e^{2 y} \zeta^{2}-E-i 0\right)^{-1} f$ satisfies the outgoing radiation condition, and $\left(-\partial_{y}{ }^{2}+e^{2 y} \zeta^{2}-E+i 0\right)^{-1} f$ the incoming radiation condition, with $\alpha=1-s$. We have also the uniform estimate

$$
\begin{gathered}
\left\|\left(-\partial_{y}{ }^{2}+e^{2 y} \zeta^{2}-z\right)^{-1}\right\|_{\mathbf{B}\left(L^{2, s} ; L^{2,-s}\right)} \leq C, \\
\left\|F( \pm y>0)\left(i \partial_{y} \pm \operatorname{Re} \sqrt{z}\right)\left(-\partial_{y}{ }^{2}+e^{2 y} \zeta^{2}-z\right)^{-1}\right\|_{\mathbf{B}\left(L^{2, s} ; L^{2, s-1}\right)} \leq C, \\
\left\|F( \pm y>0)\left(i \partial_{y} \mp \operatorname{Re} \sqrt{z}\right)\left(-\partial_{y}{ }^{2}+e^{2 y} \zeta^{2}-z\right)^{-1}\right\|_{\mathbf{B}\left(L^{2, s} ; L^{2, s-1}\right)} \leq C,
\end{gathered}
$$

for $\zeta \geq 0$ and $z \in\{0<a \leq \operatorname{Re} z \leq b<\infty, 0 \leq \pm \operatorname{Im} z \leq 1\}$, a,b being arbitrarily fixed constants, where the inequality (7.9) holds for $\operatorname{Im} z \geq 0$ and (7.10) for $\operatorname{Im} z \leq 0$.

Proof. Suppose that the uniform bound (7.8) does not hold. Then there exist $\zeta_{n} \geq 0, z_{n} \in \mathbf{C}, f_{n} \in L^{2, s}$ such that $a \leq \operatorname{Re} z_{n} \leq b, 0 \leq \operatorname{Im} z_{n} \leq 1,\left\|f_{n}\right\|_{s} \rightarrow$ $0,\left\|u_{n}\right\|_{-s}=1$, where $u_{n}=\left(-\partial_{y}{ }^{2}+e^{2 y} \zeta_{n}^{2}-z_{n}\right)^{-1} f_{n}$. If $\left\{\zeta_{n}\right\}_{n \geq 0}$ is unbounded, one can assume that $\zeta_{n} \rightarrow \infty$. By Lemma 7.1, $u_{n} \rightarrow 0$ in $L_{l o c}^{2}$. This and Lemma 7.4 imply that $u_{n} \rightarrow 0$ in $L^{2,-s}$. This is a contradiction. Therefore one can assume that $\zeta_{n} \rightarrow \zeta, z_{n} \rightarrow E$. Obviously $E \in \mathbf{R}$. By Lemmas 7.1, 7.4 and Rellich's theorem, one can choose a subsequence $u_{n_{j}}$ such that $u_{n_{j}} \rightarrow u$ in $L^{2,-s}$. Lemmas 7.1 and 7.3 imply that this $u$ is an outgoing solution of $-u^{\prime \prime}+e^{2 y} \zeta^{2} u-E u=0$. Hence $u=0$ by Lemma 7.6. This is a contradiction.

We next show the existence of the limit (7.7). For $f \in L^{2, s}$, put $u_{n}=\left(-\partial_{y}{ }^{2}+\right.$ $\left.e^{2 y} \zeta^{2}-E-i \epsilon_{n}\right)^{-1} f_{n}$ and let $\epsilon_{n} \rightarrow 0$. Arguing as above, one can choose a subsequence $\left\{u_{n_{j}}\right\}$ which converges to $u$ in $L^{2,-s}$. Moreover, this $u$ is an outgoing solution of $-u^{\prime \prime}+e^{2 y} \zeta^{2} u-E u=f$. Such a solution is unique by virtue of Lemma 7.6. Since any subsequence of $\left\{u_{n}\right\}$ contains a sub-subsequence which converges to one and the same limit, $\left\{u_{n}\right\}$ itself converges in $L^{2,-s}$ without choosing a subsequence. This completes the proof of Theorem 7.7. 


\section{Limiting Absorption Principle in $\mathbf{H}^{n}$ And $\Gamma \backslash \mathbf{H}^{n}$}

8.1 LAP in $\mathbf{H}^{n}$. In the first part of this section, we shall study

$$
H_{0}=-\partial_{y}^{2}-e^{2 y} \Delta_{x}
$$

in $L^{2}\left(\mathbf{R}^{n-1} \times \mathbf{R}^{1} ; d x d y\right)$. We pass to the Fourier transformation in $x$ and consider

$$
L_{0}\left(|\xi|^{2}\right)=-\partial_{y}^{2}+e^{2 y}|\xi|^{2}, \quad \xi \in \mathbf{R}^{n-1} .
$$

By virtue of the results in the previous section, we get the estimates uniform in $\xi$ for the resolvent of $L_{0}\left(|\xi|^{2}\right)$. Let $R_{0}(z)=\left(H_{0}-z\right)^{-1}$. For $t, s \in \mathbf{R}$, let $\mathcal{H}^{t, s}$ be the function space defined by

$$
u \in \mathcal{H}^{t, s} \Longleftrightarrow\|u\|_{t, s}=\left\|(1+|x|)^{t}(1+|y|)^{s} u(x, y)\right\|_{L^{2}\left(\mathbf{R}^{n}\right)}<\infty .
$$

$\mathcal{H}^{0, s}$ is denoted by $L^{2, s}$. The following theorem follows from Theorem 7.7.

Theorem 8.1. Let $s>1 / 2$. Then

$$
\left\|R_{0}(z)\right\|_{\mathbf{B}\left(L^{2, s} ; L^{2,-s}\right)} \leq C,
$$

for $z \in\{0<a<\operatorname{Re} z<b, \operatorname{Im} z \neq 0\}, a, b>0$ being arbitrarily fixed constants. For any $\lambda>0$, there exists the weak limit $\mathrm{w}-\lim _{\epsilon \downarrow 0} R_{0}(\lambda \pm i \epsilon) \in \mathbf{B}\left(L^{2, s} ; L^{2,-s}\right)$. Moreover we have

$$
\begin{aligned}
& F( \pm y>0)\left(i \partial_{y} \pm \sqrt{\lambda}\right) R_{0}(\lambda+i 0) \in \mathbf{B}\left(L^{2, s} ; L^{2, s-1}\right), \\
& F( \pm y>0)\left(i \partial_{y} \mp \sqrt{\lambda}\right) R_{0}(\lambda-i 0) \in \mathbf{B}\left(L^{2, s} ; L^{2, s-1}\right) .
\end{aligned}
$$

Let us study the spectral properties of $H=H_{0}+V$. We assume $V$ to satisfy (3.6), (3.7), and $V_{1}$ to be compactly supported.

Lemma 8.2. (1) For $z \in \mathbf{C} \backslash \mathbf{R}, R_{0}(z) \in \mathbf{B}\left(L^{2} ; H_{l o c}^{2}\right)$.

(2) For any $\epsilon>0$, there exists $C_{\epsilon}>0$ such that

$$
\|V u\| \leq \epsilon\left\|H_{0} u\right\|+C_{\epsilon}\|u\|, \quad \forall u \in C_{0}^{\infty}\left(\mathbf{R}^{n}\right) .
$$

Proof. The first assertion is obvious. To prove (2), we split $V_{1}=V_{1, \epsilon}+V_{2, \epsilon}$ in such a way that $\left\|V_{1, \epsilon}\right\|_{L^{p}}<\epsilon, V_{2, \epsilon} \in L^{\infty}$. Pick $\chi \in C_{0}^{\infty}\left(\mathbf{R}^{n}\right)$ such that $\chi=1$ on $\operatorname{supp} V_{1}$. Then as is well-known

$$
\left\|V_{1, \epsilon} \chi u\right\| \leq \epsilon\left\|\left(\Delta_{x}+\partial_{y}^{2}\right)(\chi u)\right\|+C_{\epsilon}\|\chi u\|, \quad \forall u \in C_{0}^{\infty}\left(\mathbf{R}^{n}\right) .
$$

To complete the proof we have only to note

$$
\left\|\left(\Delta_{x}+\partial_{y}^{2}\right)(\chi u)\right\| \leq C\left(\left\|H_{0}(\chi u)\right\|+\|\chi u\|\right)
$$

which follows from the ellipticity of $H_{0}$.

By the above lemma, $\left.H\right|_{C_{0}^{\infty}}$ is essentially self-adjoint, whose self-adjoint extension is denoted by $H$ again. By Weyl's theorem, we have

Theorem 8.3. $\sigma_{e}(H)=[0, \infty)$.

Theorem 8.4. (1) Let $0<\alpha<1 / 2$ and $\lambda>0$. If $u \in L^{2,-\alpha}$ satisfies $(H-\lambda) u=0$, then $u=0$.

(2) $\sigma_{p}(H) \cap(0, \infty)=\emptyset$.

Proof. To prove (1), we first note that by applying Theorem 9.1 with $B(t)=$ $-e^{-2 t} \Delta_{x}$, there exists $R_{1}>0$ such that $u=0$ if $y<-R_{1}$. Therefore by the unique continuation theorem (see e.g. Jerison-Kenig [15] Theorem 6.3), $u$ vanishes identically. The assertion (2) is a direct cosequence of (1). 
Definition 8.5. A solution of $u$ of the equation $(H-\lambda) u=f$ is said to satisfy the outgoing radiation condition if for some $0<\alpha<1 / 2<s$

$$
u \in L^{2,-s}, \quad F( \pm y>0)\left(i \partial_{y} \pm \sqrt{\lambda}\right) u \in L^{2,-\alpha} .
$$

The following lemma can be proved in the same way as Lemma 7.6.

Lemma 8.6. An outgoing solution of $(H-\lambda) u=f, \lambda>0$, is unique.

Similary a solution of $u$ of the equation $(H-\lambda) u=f$ is said to satisfy the incoming radiation condition if for some $0<\alpha<1 / 2<s$

$$
u \in L^{2,-s}, \quad F( \pm y>0)\left(i \partial_{y} \mp \sqrt{\lambda}\right) u \in L^{2,-\alpha} .
$$

Lemma 8.6 also holds for the incoming solution.

Lemma 8.7. Let $1<2 s \leq 1+\rho$, and $\epsilon=s-1 / 2$. Let $u \in L^{2,-s}$ satsify $\left(H-\zeta^{2}\right) u=$ $f \in L^{2, s}$, where $\operatorname{Re} \zeta>0, \operatorname{Im} \zeta \geq 0$. Then for any $\delta>0$

(1) $\left\|F( \pm y>0)\left(i \partial_{y} \pm \operatorname{Re} \zeta\right) u\right\|_{s-1} \leq C\left(\|u\|_{-s}+\|f\|_{s}\right)$,

(2) $\left.\|F(|x|+|y|>r) u\|_{\mathcal{H}^{-\delta / 2,-s}} \leq C r^{-\min (\delta, \epsilon) / 2}\left(\|u\|_{-s}+\| f\right\}_{s}\right)$,

where the costants $C$ do not depend on $r>1$ and $\zeta$ when $\zeta$ varies over $\{0<a<$ $\operatorname{Re} \zeta<b, 0<\operatorname{Im} \zeta<1\}, a, b$ being arbitrarily fixed constants.

Proof. When $V=0$, the assertion (1) follows from Lemma 7.3 by passing to the Fourier transform in $x$. When $V$ is present, we have

$$
\left\|F( \pm y>0)\left(i \partial_{y} \pm \operatorname{Re} \zeta\right) u\right\|_{s-1} \leq C\left(\|u\|_{-s}+\|f\|_{s}+\|V u\|_{s}\right) .
$$

By the decay assumption on $V$, we have $\|V u\|_{s} \leq C\|u\|_{-s}$, which proves (1).

By the same computation as in Lemma 7.4, we have

$$
\|F(|y|>r) u\|_{-s} \leq C r^{-\epsilon / 2}\left(\|u\|_{-s}+\|f\|_{s}\right) .
$$

It is easy to see

$$
\left\|F(|x|>r)\langle x\rangle^{-\delta / 2}\langle y\rangle^{-s} u\right\| \leq C r^{-\delta / 2}\|u\|_{-s} .
$$

These two inequalities yield (2).

Theorem 8.8. For $\lambda>0, \delta>0$ and $s>1 / 2$, there exists the strong limit

$$
\mathrm{s}-\lim _{\epsilon \downarrow 0} R(\lambda \pm i \epsilon) \quad \text { in } \quad \mathbf{B}\left(\mathcal{H}^{0, s} ; \mathcal{H}^{-\delta,-s}\right) .
$$

Moreover there exits the weak limt

$$
\mathrm{w}-\lim _{\epsilon \downarrow 0} R(\lambda \pm i \epsilon) \quad \text { in } \quad \mathbf{B}\left(L^{2, s} ; L^{2,-s}\right),
$$

and for $f \in L^{2, s}, R(\lambda+i 0) f$ is outgoing and $R(\lambda-i 0) f$ is incoming, with $\alpha=1-s$. For any $0<a<b<\infty$, there exists a constant $C>0$ such that

$$
\begin{gathered}
\|R(\lambda \pm i 0)\|_{\mathbf{B}\left(L^{2, s} ; L^{2,-s}\right)} \leq C, \\
\left\|F( \pm y>0)\left(i \partial_{y} \pm \sqrt{\lambda}\right) R(\lambda+i 0)\right\|_{\mathbf{B}\left(L^{2, s} ; L^{2, s-1}\right)} \leq C, \\
\left\|F( \pm y>0)\left(i \partial_{y} \mp \sqrt{\lambda}\right) R(\lambda-i 0)\right\|_{\mathbf{B}\left(L^{2, s} ; L^{2, s-1}\right)} \leq C,
\end{gathered}
$$

for $a<\lambda<b$. 
Proof. The proof of (8.4) is almost the same as Theorem 7.7. The assertion (8.5) follows from Therorem 8.1 and the resolvent equation. The assertion (8.6) is proven in the same way as Theorem 7.7 in $\mathbf{B}\left(\mathcal{H}^{0, s} ; \mathcal{H}^{-\delta,-s}\right)$, where $\delta$ is chosen sufficiently small. We then use the resolvent equation.

8.2 LAP in $\Gamma \backslash \mathbf{H}^{n}$. Let $H_{0}(\theta)$ and $H(\theta)$ be as in $\S 4$. The limiting absorption principle in $\Gamma \backslash \mathbf{H}^{n}$ is proven in the same way as above with the Fourier transformation replaced by the Fourier series. In this case we have only to use the space $L^{2, s}$ with the weight $\langle y\rangle^{s}$ only.

8.3 LAP in the exterior domain of $\Gamma \backslash \mathbf{H}^{n}$. Finally we prove LAP for $H_{e x t}(\theta)=$ $H_{0}(\theta)$ in the exterior domain $U_{e x t}=\mathbf{E} \times \mathbf{R} \backslash \bar{U}$ with Dirichlet boundary condition. The proof is the same as above if one notices the following facts.

Let $u=\left(H_{e x t}(\theta)-z\right)^{-1}$ and let $\chi(x, y) \in C^{\infty}(\mathbf{E} \times \mathbf{R})$ be such that $\chi=1$ if $|y|>R+1, \chi=0$ if $|y|<R$, where $R$ is taken so large that $U \subset\{|y|<R+1 / 2\}$. Then applying the results in $\mathbf{E} \times \mathbf{R}$ to $\chi u$, one can see that Lemma 8.7 also holds for this case. The uniqueness of the outgoing or incoming solution is proven by Theorem 9.1. We have thus shown that LAP also holds in $U_{\text {ext }}$.

\section{Growth properties of Solutions to the homogeneous Equation}

Let $X$ be a Hilbert space and consider the following differential equation for an $X$-valued function $u(t)$ of $t>0$ :

$$
-u^{\prime \prime}(t)+B(t) u(t)+V(t) u(t)-E u(t)=f(t),
$$

$E$ being a positive constant. We assume that

(A-1) For each fixed $t, B(t)$ is non-negative, self-adjoint on $X$ with domain $D(B(t))=$ $D$ independent of $t$. For each $x \in D$, the map $(0, \infty) \ni t \rightarrow B(t) x \in X$ is $C^{1}$ and there exists $t_{0} \geq 0$ such that

$$
t \frac{d B(t)}{d t}+B(t) \leq 0, \quad \forall t \geq t_{0}
$$

(A-2) For each $t, V(t)$ is bounded, self-adjoint on $X$. Further $V(t)=V_{L}(t)+V_{S}(t)$, where

$$
V_{L}(t) \in C^{1}((0, \infty) ; \mathbf{B}(X)), \quad V_{S}(t) \in C^{0}((0, \infty) ; \mathbf{B}(X)),
$$

and there exist $C, \epsilon>0$ such that

$$
\begin{aligned}
& \left\|V_{L}(t)\right\| \leq C(1+t)^{-\epsilon}, \quad\left\|\frac{d V_{L}(t)}{d t}\right\|+\left\|V_{S}(t)\right\| \leq C(1+t)^{-1-\epsilon}, \quad \forall t \geq 0 . \\
& \|f(t)\|_{X} \leq C(1+t)^{-1-\epsilon}\|u(t)\|_{X}, \quad \forall t \geq 0 .
\end{aligned}
$$

Theorem 9.1. Assume (A-1), (A-2), (A-3). If

$$
\liminf _{t \rightarrow \infty}\left(\left\|u^{\prime}(t)\right\|_{X}+\|u(t)\|_{X}\right)=0
$$

there exists $t_{1}>0$ such that $u(t)=0$ for $\forall t>t_{1}$.

This theorem is proved in the same way as in pp. 29 - 35 of [30] with a slight modification. By virtue of our assumption (A-1), we have

$$
-\left(\frac{d B(t)}{d t} v, v\right)_{X} \geq \frac{1}{t}(B(t) v, v)_{X} .
$$


We use the notation of [30] and follow his computation using (9.1). Then the inequality (3.5) in [30] reads

$$
\frac{d}{d r}(K v) \geq-2 c_{2}(1+r)^{-2 \delta}|v|_{X}\left|v^{\prime}\right|_{X}+\frac{1}{r}(B v, v)_{X}-\left(C_{0}^{\prime} v, v\right)_{X}
$$

Therefore his Lemma 3.2 also hlods in our case. His formula (3.13) reads

$$
\begin{aligned}
\frac{d}{d r}(N v) & =\left(4 m r^{1-\mu}+1\right)\left|w^{\prime}\right|_{X}^{2}+\left\{k^{2}+(1-2 \mu) r^{-2 \mu}\left(m^{2}-\log r\right)-r^{-2 \mu}\right\}|w|_{X}^{2} \\
& -\left(\left(C_{0}+r C_{0}^{\prime}\right) w, w\right)_{X}+2 r e^{d} \operatorname{Re}\left(C_{1} v-f, w^{\prime}\right)_{X} \\
& -2\left(\mu m r^{-\mu}+r^{1-2 \mu} \log r\right) \operatorname{Re}\left(w, w^{\prime}\right)_{X}-\left(\left(r \frac{d B(r)}{d r}+B(r)\right) w, w\right)_{X} .
\end{aligned}
$$

Dropping the last term, we see that his inequality (3.14) also holds in our case. The remaining argument is entirely the same.

\section{INVERSE SCATTERING AT THE CUSP}

Let $\mathcal{M}$ be an $n$-dimesional connected Riemannian manifold. Suppose $\mathcal{M}$ consists of two parts : $\mathcal{M}=\mathcal{M}_{0} \cup \mathcal{M}_{\infty}$, where $\overline{\mathcal{M}_{0}}$ is compact, and $\mathcal{M}_{\infty}$ is diffeomorphic to $\mathbf{E} \times(1, \infty), \mathbf{E}=\Gamma \backslash \mathbf{R}^{n-1}, \Gamma$ being a lattice of rank $n-1$ in $\mathbf{R}^{n-1}$. We assume that the Riemannian metric $g$ of $\mathcal{M}$, when restricted to $\mathcal{M}_{\infty}$, takes the following form :

$$
\left.g\right|_{\mathcal{M}_{\infty}}=(d y)^{2}+e^{-2 y}(d x)^{2}
$$

where $y \in(1, \infty)$ and $(d x)^{2}$ is the flat metric on $\mathbf{E}$. We consider the Schrödinger operator

$$
H=-\Delta_{g}+A,
$$

where $A$ is a formally self-adjoint 2 nd order differential operator. We assume that for $j=1,2$ the coefficients of $j$-th covariant derivatives are in $C^{j}$, and that the multiplication operator term is bounded. Moreover we assume the following.

The supports of the coefficients of $A$ are contained in a bounded contractible set in $\mathcal{M}$.

We impose a suitable boundary condition on $\partial \mathcal{M}$ (if it exists) so that $H$ is selfadjoint. It can be shown that

Theorem 10.1. (1) $\sigma_{e}(H)=\left[(n-1)^{2} / 4, \infty\right)$.

(2) $\sigma_{p}(H) \cap\left((n-1)^{2} / 4, \infty\right)$ is discrete.

We fix a point $P_{0} \in \mathcal{M}$ arbitrarily and let $\rho$ be the geodesic distance from $P_{0}$. For $s \in \mathbf{R}$, we define the function space $L^{2, s}$ by

$$
f \in L^{2, s} \Longleftrightarrow\|f\|_{s}^{2}=\int_{\mathcal{M}}\langle\log (1+\rho)\rangle^{2 s}|f|^{2} d \mathcal{M}
$$

A solution $u$ of the equation $(H-\lambda) u=f$ is said to satisfy the outgoing (or incoming) radiation condition if

$$
u \in L^{2,-s}, \quad F(\rho>1)\left(\frac{\partial}{\partial r} \mp i k(\lambda)\right) u \in L^{2,-\alpha}, \quad k(\lambda)=\sqrt{\lambda-\frac{(n-1)^{2}}{4}}
$$

for some $0<\alpha<1 / 2<s$, where $r=\log \rho$ and we choose the - sign for outgoing and the + sign for incoming radiation condition. Let $R(z)=(H-z)^{-1}$. 
Theorem 10.2. Let I be a compact interval in $\left((n-1)^{2} / 4, \infty\right) \backslash \sigma_{p}(H)$. Then for $1 / 2<s<1$, there exists a constant $C>0$ such that for $\lambda \in I$

$$
\begin{gathered}
\|R(\lambda \pm i 0) f\|_{-s} \leq C\|f\|_{s}, \\
\left\|F(\rho>1)\left(\frac{\partial}{\partial r} \mp i k(\lambda)\right) R(\lambda \pm i 0) f\right\|_{s-1} \leq C\|f\|_{s} .
\end{gathered}
$$

For $\lambda \in\left((n-1)^{2} / 4, \infty\right) \backslash \sigma_{p}(H)$, the solution of the equation $(H-\lambda) u=f$ satisfying the outgoing or incoming radiation condition is unique.

Sketch of the proof. We use the coordinates $(x, y)$ in $\S 4$. Let $\chi(y) \in C^{\infty}$ be such that $\chi(y)=1\left(y>R_{0}+1\right), \chi(y)=0\left(y<R_{0}\right)$. By taking $R_{0}$ large enough, $\chi(y) R(z) f$ satisfies

$$
\left(-\partial_{y}^{2}-e^{2 y} \Delta_{x}-z+\frac{(n-1)^{2}}{4}\right) \chi(y) R(z) f=\chi(y) f-\left[\partial_{y}^{2}, \chi(y)\right] R(z) f .
$$

Therefore one can apply the results in $\S 8$ to $\chi(y) R(z) f$. The remaining part is treated as compact perturbation. The first part of the theorem is then reduced to the second half.

To prove the second part, let $u$ be the outgoing solution of the equation $(H-$ $\lambda) u=0$. Pick $\chi_{R}(y) \in C^{\infty}(\mathbf{R})$ such that $\chi_{R}(y)=1(y<R), \chi_{R}(y)=0(y>R+1)$ and put

$$
\tilde{\chi}_{R}(y)=\int_{y}^{\infty} \chi_{R}(t)\langle t\rangle^{-2 \alpha} d t \in C_{0}^{\infty}((1, \infty)),
$$

Taking the imaginary part of

$$
0=\left(\left(-\partial_{y}^{2}-e^{2 y} \Delta_{x}-k(\lambda)^{2}\right) u, \widetilde{\chi}_{R} u\right),
$$

we have $\operatorname{Im}\left(\partial_{y} u, \widetilde{\chi}_{R}^{\prime}(y) u\right)=0$. Hence

$$
\operatorname{Im}\left(\partial_{u} y, \chi_{R}(y)\langle y\rangle^{-2 \alpha} u\right)=0,
$$

Since $\partial_{y} u-i k(\lambda) u \in L^{2,-\alpha}$, we then see that $\left\|\sqrt{\chi_{R}(y)} u\right\|_{-\alpha} \leq C$, with $C$ independent of $R>0$. Letting $R \rightarrow \infty$, we get $u \in L^{2,-\alpha}$.

We next expand $u$ into a Fourier series in $x$. For $\gamma^{*} \in \Gamma^{*}, \hat{u}\left(\gamma^{*}, y\right)$ satisfies the equation

$$
\left(-\partial_{y}^{2}+e^{2 y}\left|\gamma^{*}\right|^{2}-k(\lambda)^{2}\right) \hat{u}\left(\gamma^{*}, y\right)=0
$$

for large $y$. Comparing its behavior with that of modified Bessel functions, we see that $\hat{u}\left(\gamma^{*}, y\right) \in L^{2}$. Therefore $u$ is an $L^{2}$-eigenfunction of $H$, hence it vanishes identically. $\diamond$.

We next introduce the exponentially growing solution at the cusp. Let $\mathcal{F}_{0 c}(\lambda)$ be as in $\S 4$. Pick $\chi(y) \in C^{\infty}$ such that $\chi(y)=1(y>3), \chi(y)=0(y<2)$ and put

$$
\widetilde{\mathcal{F}}_{0 c}(\lambda)^{*}=\chi(y) \mathcal{F}_{0 c}^{( \pm)}\left(k(\lambda)^{2}\right)^{*}-R_{0}(\lambda \pm i 0)\left(-\Delta_{g}-\lambda\right) \chi(y) \mathcal{F}_{0 c}^{( \pm)}\left(k(\lambda)^{2}\right)^{*},
$$

where $R_{0}(z)=\left(-\Delta_{g}-z\right)^{-1}$. Here we must assume that $\lambda \notin \sigma_{p}\left(-\Delta_{g}\right)$. Then for $\varphi \in L^{2}(\mathbf{E})$

$$
\left(-\Delta_{g}-\lambda\right) \widetilde{\mathcal{F}}_{0 c}^{( \pm)}(\lambda)^{*} \varphi=0
$$

and $\widetilde{\mathcal{F}}_{0 c}^{( \pm)}(\lambda)^{*} \varphi$ is exponentially growing at the cusp. We let

$$
u=\widetilde{\mathcal{F}}_{0 c}^{(-)}(\lambda)^{*} \varphi-R(\lambda+i 0)\left(A \widetilde{\mathcal{F}}_{0 c}^{(-)}(\lambda)^{*} \varphi\right) .
$$

Then $u$ satisfies

$$
(H-\lambda) u=0 .
$$


Moreover we have

$$
F(\rho>1)\left(\frac{\partial}{\partial r}-i k(\lambda)\right)\left(u-\widetilde{\mathcal{F}}_{0 c}(\lambda)^{*} \varphi\right) \in L^{2,-\alpha}
$$

for some $0<\alpha<1 / 2$. Such a solution is unique by virtue of Theorem 10.2. As in Theorem 4.11, we have the following asymptotic expansion.

Theorem 10.3. For $\varphi \in L^{2}(\mathbf{E})$, we have as $\rho \rightarrow \infty$

$$
\begin{gathered}
\left\langle e^{-i \gamma^{*} \cdot x}, u-\widetilde{\mathcal{F}}_{0 c}^{(-)}(\lambda)^{*} \varphi\right\rangle \sim-\sqrt{\frac{\pi}{2}}\left|\gamma^{*}\right|^{-1 / 2} \rho^{-1 / 2} e^{-\left|\gamma^{*}\right| \rho} \mu_{\gamma^{*}} \mathbf{A}_{c \gamma^{*}}(\lambda) \varphi \quad\left(\gamma^{*} \neq 0\right) \\
\left\langle e^{-i \gamma^{*} \cdot x}, u-\widetilde{\mathcal{F}}_{0 c}^{(-)}(\lambda)^{*} \varphi\right\rangle \sim i \frac{\sqrt{2 \pi}}{k(\lambda)} \rho^{i y k(\lambda)} \mathbf{A}_{c \gamma^{*}}(\lambda) \varphi \quad\left(\gamma^{*}=0\right),
\end{gathered}
$$

$\left\{\mathbf{A}_{c \gamma^{*}}(\lambda)\right\}_{\gamma^{*} \in \Gamma^{*}}$ being bounded in $\mathbf{B}\left(L^{2}(\mathbf{E}), \mathbf{C}\right)$.

As in $\S 4$

$$
\mathbf{A}_{c}(\lambda)=\sum_{\gamma^{*} \in \Gamma^{*}} e^{i \gamma^{*} \cdot x} \mathbf{A}_{c \gamma^{*}}(\lambda)
$$

defines the scattering amplitude at the cusp.

Take a bounded contractible domain $\Omega \subset \mathcal{M}$ such that $A=0$ outside $\Omega$, and define the D-N map $\Lambda(A)$ for $H_{D}=-\Delta_{g}+A$ in $\Omega$ with Dirichlet boundary condition. Then by the same arguments as in $\S 6$, we have

Theorem 10.4. Suppose $\lambda \notin \sigma_{p}(H) \cup \sigma_{p}\left(-\Delta_{g}\right) \cup \sigma_{p}\left(H_{D}\right)$. Then the scattering amplitude at the cusp $\mathbf{A}_{c}(\lambda)$ and the $D-N$ map $\Lambda(A)$ determine each other.

With the aid of Theorem 10.4, one can argue the reconstruction of the local perturbation of the metric. Let $\mathcal{M}$ be an $n$-dimensional hyperbolic manifold with a cusp. Take a bounded contractible domain $\Omega$ in $\mathcal{M}$. If $n \geq 3$, the conformal deformation of the metric in $\Omega$ can be reconstructed from the scattering amplitude at the cusp by using the result of Sylvester-Uhlmann [36] and Nachman [25]. If $n=2$, one can deal with the general perturbation of the metric and reconstruct $\sqrt{\operatorname{det}\left(g_{i j}\right)} g^{i j}$ by virtue of the result of Nachman [26]. For two metrics $g$ and $\bar{g}$, $\sqrt{\operatorname{det}\left(g_{i j}\right)} g^{i j}=\sqrt{\operatorname{det}\left(\bar{g}_{i j}\right)} \bar{g}^{i j}$ is equivalent to that $g$ and $\bar{g}$ are conformal. Therefore the coincidence of the scattering amplitudes assocaited with $g$ and $\bar{g}$ is equivalent to the conformality of $g$ and $\bar{g}$ (see also [23]). One can also treat the case of many cusps.

\section{REFERENCES}

[1] S. Agmon, Spectral theory of Schrödinger operators on Euclidean and non-Euclidean spaces, Comm. Pure Appl. Math. 39, Supplement (1986), S 3 - S 16.

[2] M. I. Belishev and Y. V. Kurylev, To the reconstruction of a Riemannian manifold via its boundary spectral data (BC method), Commun. P. D. E. 17 (1992), 767-804.

[3] R. Brown and G. Uhlmann, Uniqueness in the inverse conductivity problem for nonsmooth conductivities in two dimensions, Commun. P. D. E. 22 (1997), 1009-1027.

[4] G. Eskin, The inverse scatering problem in two dimensions at fixed energy, Commun. in P. D. E. 26 (2001), 1055-1090.

[5] G. Eskin and J. Ralston, Inverse scattering problem for the Schrödinger equation with magnetic potential at a fixed energy, Commun. Math. Phys. 173 (1995), 199-224.

[6] L. D. Faddeev, Inverse problems of quantum scattering theory, J. Sov. Math. 5 (1976), 334396. 
[7] R. Froese and P. Hislop, Spectral analysis of second-order elliptic operators on non-compact manifolds, Duke Math. J. 58 (1989), 103-129.

[8] P. D. Hislop, The geometry and spectra of hyperbolic manifolds, Proc. Indian Acad. Sci. (Math. Sci.), 104 (1994), 715-776.

[9] T. Ikebe and Y. Saito, Limiting absorption method and absolute continuity for the Schrödinger operator, J. Math. Kyoto Univ. 12 (1972), 513-542.

[10] V. Isakov and A. Nachman, Global uniqueness for a two-dimensional semilinear elliptic inverse problem, Trans. A. M. S. 347 (1995), 3375-3390.

[11] V. Isakov and Z. Sun, The inverse scattering at fixed energies in two dimensions, Indiana Univ. Math. J. 44 (1995), 883-896.

[12] H. Isozaki, Multi-dimensional inverse scattering theory, Analytic Extension Formulas and Their Applications, pp. 123-167, eds. S. Saito et al, Kluwer Academic Publishers (2001).

[13] H. Isozaki, Inverse problems and hyperbolic manifolds, Contemporary Mathematics 348, Inverse Problems and Spectral Theory, ed. H. Isozaki, A. M. S., Providence, Rhode Island (2004), 181-197.

[14] H. Isozaki and G. Uhlmann, Hyperbolic geometry and local Dirichlet-Neumann map, Adv. in Math. 188 (2004), 294-314.

[15] D. Jerison and C. E. Kenig, Unique continuation and absence of positive eigenvalues for Schrödinger operators, Ann. of Math. 121 (1985), 463-494.

[16] H. Kang, A uniqueness theorem for an inverse boundary value problem in two dimensions, $J$. Math. Anal. Appl. 270 (2002), 291-302.

[17] H. Kang and G. Uhlmann, Inverse problems for the Pauli Hamiltonian in two dimensions, $J$. Fourier Anal. Appl. 10 (2004), 201-215.

[18] A. Katchalov, Y. Kurylev and M. Lassas, Inverse Boundary Spectral Problems, Chapman \& Hall (2001).

[19] G. M. Khenkin and R. G. Novikov, The $\bar{\partial}$-equation in the multi-dimensional inverse scattering problem, Russian Math. Surveys 42 (1987), 109-180.

[20] S. T. Kuroda, Scattering theory for differential operators, I, II, J. Math. Soc. Japan, 25 (1972), 75-104, 222-234.

[21] P. D. Lax and R. S. Phillips, Scattering Theory for Automorphic Functions, Princeton University Press, Princeton (1976).

[22] N. N. Lebedev, Special Functions and Their Applications, Dover (1972).

[23] J. Lee and G. Uhlmann, Determining anisotropic real-analytic conductivity by boundary measurements, Comm. Pure Appl. Math. 42 (1989), 1097-1112.

[24] R. Melrose, Geometric Scattering Theory, Cambridge, Cambridge University Press (1995).

[25] A. Nachman, Reconstruction from boundary measurements, Ann. of Math. 128 (1988), 531576.

[26] A. Nachman, Global uniqueness for a two dimensional inverse boundary value problem, Ann. of Math. 143 (1996), 71-96.

[27] R. G. Novikov, The inverse scattering problem on a fixed energy level for the two-dimensional Schrödinger operator, J. Funct. Anal. 103 (1992), 409-463.

[28] P. A. Perry, The Laplace operator on a hyperbolic manifold $I$. Spectral and scattering theory, J. Funct. Anal. 75 (1987), 161-187.

[29] M. Reed and B. Simon, Methods of Modern Mathematical Physics, Vol 1 (1980), Vol 4 (1978), Academic Press, San Diego.

[30] Y. Saito, Spectral Representations for Schrödinger Operators with Long-Range Potentials, Lecture Notes in Math. 727, Springer-Verlag, Berlin-Heidelberg-New York (1979).

[31] Z. Sun, On an inverse boundary value problem in two dimensions, Commun. P. D. E. 14 (1989), 1101-1113.

[32] Z. Sun and G. Uhlmann, Generic uniqueness for an inverse boundary value problem, Duke Math. J. 62 (1991), 131-155.

[33] Z. Sun and G. Uhlmann, Recovery of singularities for formally determined problems, Commun. in Math. Phys. 153 (1993), 431-445.

[34] J. Sylvester, An anisotropic inverse boundary problem, Comm. Pure Appl. Math. 43 (1990), 201-233.

[35] J. Sylvester and G. Uhlmann, A uniqueness theorem for an inverse boundary value problem in electrical prospection, Comm. Pure Appl. Math. 39 (1986), 91-112. 
[36] J. Sylvester and G. Uhlmann, A global uniqueness theorem for an inverse boundary value problem, Ann. of Math. 125 (1987), 153-169.

[37] A. Terras, Harmonic Analysis on Symmetric Spaces and Applications I, Springer-Verlag, Berlin-Heidelberg-New York (1985).

[38] M. Tsuji, Potential Theory in Modern Function Theory, Maruzen, Tokyo (1959)

[39] G. N. Watson, A Treatise on the Theory of Bessel Functions, Cambridge University Press (1980).

Graduate School of Pure and Applied Sciences, University of Tsukuba, Tsukuba, 305-8571, JAPAN

E-mail address: isozakih@math.tsukuba.ac.jp 